\title{
Inferring Earthquake Ground-Motion Fields with Bayesian Networks
}

\author{
by Pierre Gehl, John Douglas, and Dina D'Ayala
}

\begin{abstract}
Bayesian networks (BNs) have the ability to perform inference on uncertain variables given evidence on observed quantities, which makes them relevant mathematical tools for the updating of ground-motion fields based on strong-motion records or macroseismic observations. Therefore, the present article investigates the use of BN models of spatially correlated Gaussian random fields as an accurate and scalable method for the generation of ground-motion maps. The proposed BN model is based on continuous Gaussian variables, as opposed to discrete variables as in previous formulations, and it is built to account for cross-correlated ground-motion parameters as well as macroseismic observations. This approach is validated with respect to the analytical solution (i.e., conditional multivariate normal distributions), and it is also compared with the U.S. Geological Survey ShakeMap method, thus demonstrating a better ability to model jointly the interevent and intraevent error terms of ground-motion models. The scalability of the approach, that is, its capacity to be applied to large grids, is ensured by a grid subdivision strategy, which appears to be computationally efficient and accurate within an error rate of a fraction of percent. Finally, the BN implementation is demonstrated on a real-world example (the 2016 $M_{\mathrm{w}} 6.2$ Kumamoto, Japan, foreshock), where vector-valued shake maps of crosscorrelated intensity measures are generated, along with the integration of macroseismic observations.
\end{abstract}

\section{Introduction}

Over the past decade, rapid loss assessment following earthquakes has emerged as a crucial research topic, with the objective of providing emergency responders and critical facility operators with accurate estimates of intensity levels or probable damage across the affected area (e.g., Wald et al., 2008; Erdik et al., 2011). For instance, at the hazard level, updating the spatially distributed ground-motion field, or ground-motion map, is achieved by combining estimates from ground-motion prediction equations (GMPEs) and field observations (Wald et al., 2005; Worden et al., 2010). A comparison of the most common statistical techniques is provided by Douglas (2007) for the 2004 Les Saintes (Guadeloupe, France) earthquake. Worden et al. (2010) also provide valuable insights into pending issues, namely the treatment of uncertainties near the observations, the quality of the estimates for poorly observed events, and the computation of joint distributions for correlated intensity measures (IMs). A rigorous probabilistic analysis of the relation between macroseismic intensity and peak ground acceleration (PGA) has been proposed by Ebel and Wald (2003), but without accounting for correlation between spatially distributed ground motions.

The inference abilities of Bayesian networks (BNs) appear to be appealing for such a problem because they use observations as evidence to update directly the prior distri- butions of various variables, such as estimates from GMPEs or the damage distribution (Jaiswal et al., 2011). The application of BNs to earthquake engineering has been formalized by Bensi et al. (2011a) for the analysis of infrastructure systems of interdependent elements, which requires the estimation of statistics for joint events over spatially distributed assets. Besides forward risk analyses (Bensi et al., 2013), BNs may also be used for the backward analysis of a system when a partial knowledge of losses is available immediately after an earthquake (e.g., Pozzi and Der Kiureghian, 2013; Gehl et al., 2017). Most proposed BN formulations are, however, hampered by scalability and computational issues, which complicate their application to real-world systems (Cavalieri et al., 2017).

Therefore, the present article builds upon the original BN approach by Bensi et al. (2011a), while applying the Bayesian framework to the ground-motion assessment part only. It is expected that the removal of the variables related to damage and system performance estimation will greatly reduce the computational difficulties, mostly by enabling the use of continuous Gaussian BNs, as opposed to the discrete BNs used in previous studies. Moreover, the BN formulation is augmented with additional variables representing secondary cross-correlated IMs and even macroseismic intensities, so that Bayesian updating can be performed 
with diverse sources of field observations. The proposed developments pursue multiple objectives: (1) to demonstrate the accuracy of the BN approach for the generation of ground-motion maps, which is a prerequisite before complete BNs enabling loss estimation may be used in a decision support system; (2) to verify the feasibility and scalability of the BN approach for large spatial grids in the case of realworld earthquakes; and (3) to investigate the potential benefits that can be gained from inferring ground-motion fields with a BN, especially in terms of uncertainty treatment (e.g., joint updating of interevent and intraevent error terms) and the generation of maps for vector-valued IMs. The underlying equations necessary to model the formulation of the proposed $\mathrm{BN}$ are presented in the Demonstration of the Bayesian Network Approach section, which also contains a comparative analysis of the $\mathrm{BN}$ approach with respect to the well-established ShakeMap algorithm (Worden and Wald, 2016). The scalability issue is addressed in the Computational Performance section, in which a subgrid division strategy is investigated to ensure the stability of the BN. Finally, the Application to the $\mathrm{M}_{\mathrm{w}} 6.2$ Kumamato Earthquake (14 April 2016) section applies the BN approach to a specific event, the 14 April $2016 M_{\mathrm{w}} 6.2$ earthquake near Kumamoto, Japan, thus providing an opportunity to demonstrate the implementation of the $\mathrm{BN}$ on an actual earthquake and to analyze the information gain when considering multiple cross-correlated IMs.

\section{Demonstration of the Bayesian Network Approach}

This section provides details on the construction of the $\mathrm{BN}$ and an investigation of its validity with respect to other methods for the generation of ground-motion maps.

Proposed Approach for the Construction of the Bayesian Network

The distribution of a given strong-motion parameter or IM over a given geographical grid is usually estimated from the following variables (Crowley and Bommer, 2006):

- $M_{\mathrm{w}}$, the moment magnitude of the earthquake event;

- Epi, the location of the epicenter of the earthquake, if a point-source event is assumed, or the rupture location and extent for finite-fault scenarios;

- other parameters such as the faulting mechanism, the fault geometry, and the depth to top of rupture, depending on the specific GMPE that is used;

- $X_{i}$, the logarithm of the median estimate of the IM at the grid point $i$, as predicted by the selected GMPE (i.e., $X_{i}=\ln \overline{\mathrm{IM}_{i}}$ );

- $\eta$, the interevent (or between-event) error term from the GMPE;

- $\zeta$, the intraevent (or within-event) error term from the GMPE; and
- $Y_{i}$, the logarithm of the IM distribution at the grid point $i$, accounting for the aleatory variability generated by the GMPE error terms (i.e., $Y_{i}=\ln \mathrm{IM}_{i}$ ).

It should be noted that $X_{i}, \eta$, and $\zeta$ depend on the GMPE chosen and hence they are a function of its database, functional form, and the technique used for its derivation. There can be considerable differences in these variables depending on the GMPE chosen (epistemic uncertainty), particularly at the edges of their applicability (e.g., large magnitudes and close source-to-site distances; Douglas and Edwards, 2016). When there are few observations, these differences would map to large differences in the ground-motion field estimates. However, when dense observations exist, the BN method presented below would lead to these differences being reduced, and the choice of the original GMPE would then be less important.

According to Park et al. (2007) and Crowley et al. (2008a), the same interevent variability should be applied to all grid points within a given earthquake scenario, whereas the joint distribution of the intraevent term should follow the spatial correlation among grid points. As shown by Bensi et al. (2011b), representing the dependency among grid points is facilitated by a Cholesky factorization of the correlation matrix. Let us assume a grid of $n$ points, in which the variability of the intraevent term is represented by a correlated Gaussian random field defined by standard normal variables $Z_{i}$ at grid points $i$. The proposed decomposition is then performed as follows:

$$
\mathbf{Z}=\mathbf{T U}
$$

in which the $n \times n$ transformation matrix $\mathbf{T}$ is a lower triangular matrix obtained through a Cholesky factorization, so that $\mathbf{R}=\mathbf{T T}^{T}$, with $\mathbf{R}$ being the correlation matrix of each couple of the grid points. The $n \times 1$ vector $\mathbf{U}$ represents the standard normal variables, which are statistically independent from each other and are used to model the variation in the correlation among the grid points. The correlation matrix $\mathbf{R}$ is built thanks to a spatial correlation model, such as the one proposed by Jayaram and Baker (2009), in which the correlation coefficient $\rho_{i j}$ between the ground-motion parameters at two sites $i$ and $j$ is expressed as

$$
\rho_{i j}=\exp \left(-\frac{3 r_{i j}}{b}\right)
$$

in which $r_{i j}$ is the distance between the sites and $b$ is the correlation distance, assumed here to be equal to $13.5 \mathrm{~km}$ for PGA, which is consistent with recent studies on spatial correlation (e.g., Jayaram and Baker, 2009; Esposito and Iervolino, 2011).

When the ground-motion field is generated to estimate losses for various types of assets, such as an infrastructure system, the method may need to provide estimates for more than one IM, depending on the type of fragility models used. 


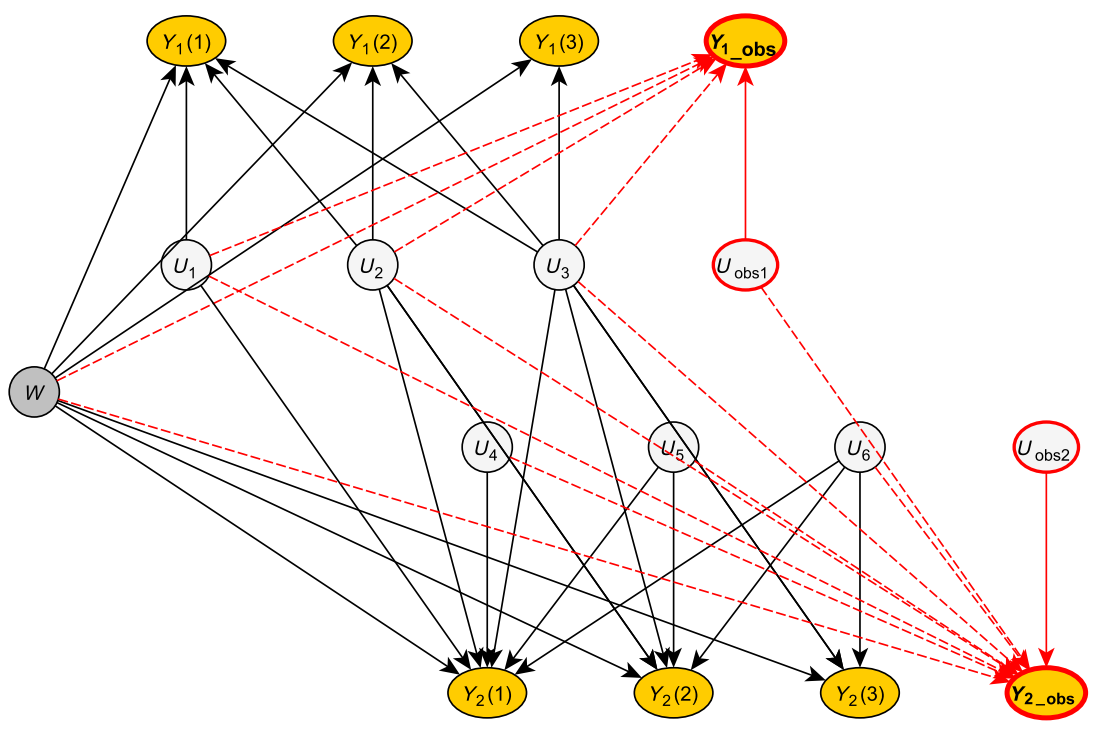

Figure 1. Example of a Bayesian network $(\mathrm{BN})$ structure for the prediction of the spatial ground-motion distribution for three grid points $\left(Y_{1}\right.$ represents the principal intensity measure [IM] and $Y_{2}$ the secondary IM). The nodes $Y_{1 \_ \text {obs }}$ and $Y_{2 \_ \text {obs }}$ in bold represent an observation (i.e., evidence) of the two IMs at a given location. The color version of this figure is available only in the electronic edition.

Therefore, the cross correlation between the IMs of interest must be taken into account when computing their joint distribution. When modeling a ground-motion field of $n_{\mathrm{IM}}$ cross-correlated IMs over $n$ sites, the corresponding correlation matrix must be of the order of $n_{\mathrm{IM}} \times n$, if it is directly used in equation (1) (Weatherill et al., 2014). Therefore, because this matrix can rapidly become large, Weatherill et al. (2014) advocate the use of a sequential simulation method, which first generates a field of primary IMs, represented by the correlated vector $\mathbf{Z}_{1}$ of standard normal variables. Then,
The proposed BN structure corresponding to the above detailed variables is presented in Figure 1. The selected GMPE directly establishes a deterministic relationship between $M_{\mathrm{w}}$, Epi, and $\overline{\mathrm{IM}_{i}}$ at site $i$. For this study, we assume here that the magnitude and epicenter are known for a given earthquake. Hence, the BN structure may be greatly simplified with respect to the original $\mathrm{BN}$ formulation by Bensi et al. (2011a): only the variables that have a probabilistic dependency between each other are displayed, namely $Y_{i}, W$, and $U_{i}$ (representing $\eta$ and $\zeta$ ).

Because all the $\mathrm{BN}$ variables may be expressed as normal distributions (i.e., $W$ and $U_{i}$ are standard normal variables, and the normal distribution of the parameters $Y_{i}$ is a very common assumption in ground-motion prediction), it is possible to define the BN in Figure 1 as a Gaussian Bayesian network (GBN), as introduced by Murphy (2002). In this case, all BN nodes become continuous normal variables with parameters expressed as a linear combination of the values of the parent nodes. In the proposed example, the root nodes $U_{i}$ and $W$ are defined by a marginal distribution (i.e., normal probability density function represented by $N$ ):

$$
\left\{\begin{array}{l}
p\left(U_{i}\right)=N(0,1) \\
p(W)=N(0,1) .
\end{array}\right.
$$

Meanwhile, the conditional distribution of the child nodes $Y_{i}$ (i.e., $Y_{1, i}$ as primary IM and $Y_{2, i}$ as secondary IM) is expressed as follows:

$$
\left\{\begin{array}{l}
p\left(Y_{1, i} \mid \mathbf{U}_{1}, W\right)=N\left(X_{1, i}+\sigma_{\varsigma} \sum_{j=1}^{n} t_{i j}^{(1)} U_{j}+\sigma_{\eta} W, \varepsilon^{2}\right) \\
p\left(Y_{2, i} \mid \mathbf{U}_{1}, \mathbf{U}_{2}, W\right)=N\left(X_{2, i}+\sigma_{\varsigma} \rho_{12} \sum_{j=1}^{n} t_{i j}^{(2)} U_{j}+\sigma_{\varsigma} \sqrt{1-\rho_{12}^{2}} \sum_{j=1}^{n} t_{i j}^{(2)} U_{n+j}+\sigma_{\eta} W, \varepsilon^{2}\right),
\end{array}\right.
$$

the field of secondary IMs, represented by the correlated vector $\mathbf{Z}_{2}$ of standard normal variables, is conditioned upon the distribution of the primary IMs. These variables may then be expressed as follows:

$$
\left\{\begin{array}{l}
\mathbf{Z}_{1}=\mathbf{T}_{1} \mathbf{U}_{1} \\
\mathbf{Z}_{2}=\mathbf{T}_{2}\left(\rho_{12} \mathbf{U}_{1}+\sqrt{1-\rho_{12}^{2}} \mathbf{U}_{2}\right)
\end{array}\right.
$$

(Oliver, 2003), in which $\mathbf{T}_{1}$ and $\mathbf{T}_{2}$ are the $n \times n$ triangular transformation matrices that are factorized from the correlation matrices $\mathbf{R}_{1}$ and $\mathbf{R}_{2}$, for the primary and secondary IMs, respectively. $\mathbf{U}_{1}$ and $\mathbf{U}_{2}$ are $n \times 1$ vectors of independent standard normal variables. Finally, $\rho_{12}$ represents the crossIM correlation coefficient between the primary and the secondary IMs. in which $\sigma_{\zeta}$ and $\sigma_{\eta}$, respectively, represent the standard deviations of the intraevent and interevent error terms, which are provided by the GMPE. The coefficients $t_{i j}^{(1)}$ and $t_{i j}^{(2)}$, respectively, represent the elements of the transformation matrices $\mathbf{T}_{1}$ and $\mathbf{T}_{2}$. If it is assumed that $Y_{1, i}$ and $Y_{2, i}$ are completely determined by knowledge of $U_{i}$ and $W$, a value close to zero has to be assumed for their standard deviation $\varepsilon$ to achieve convergence. A standard deviation corresponding to the record-to-record variability may also be assigned, if it is specified by the GMPE.

In the case that an earthquake event is recorded by a set of accelerometers, the recorded ground motions may be used to update the predicted ground-motion field. Thanks to the proposed Bayesian approach, an inference can be performed through the $U_{i}$ and $W$ variables, which are used to pass the 
(a)

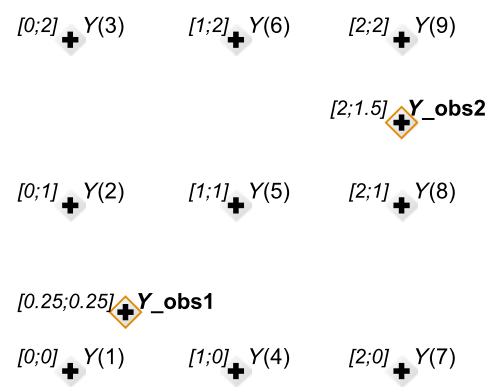

(b)

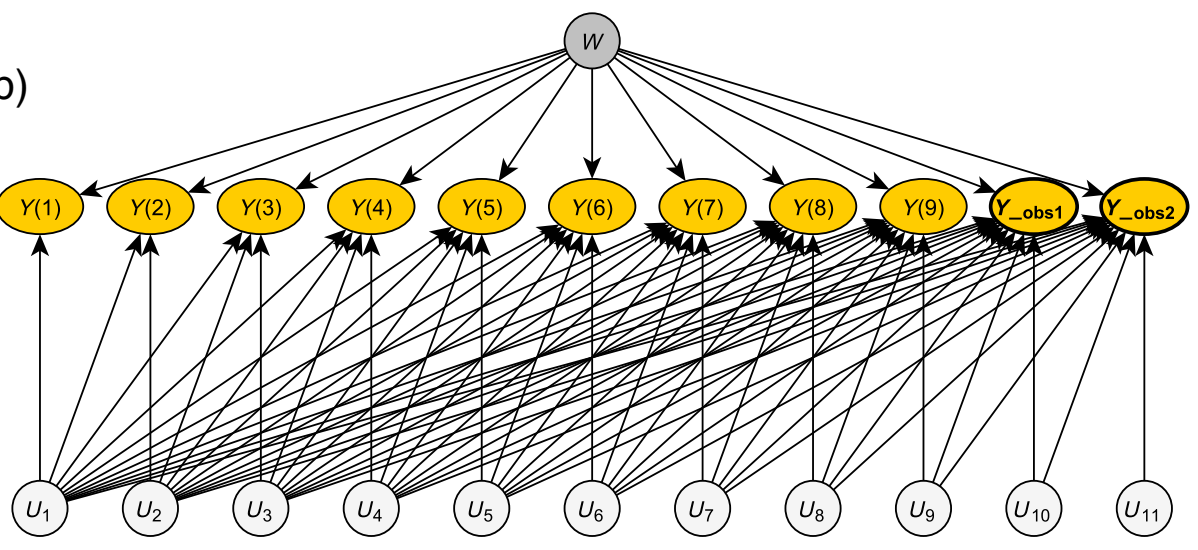

Figure 2. (a) Spatial configuration of the synthetic example used in the demonstration and (b) corresponding BN formulation. The color version of this figure is available only in the electronic edition.

message to the neighboring sites. To this end, the original BN formulation is augmented with the addition of the nodes representing the observed ground motions (i.e., nodes in bold in Fig. 1), which are then used as evidence for the Bayesian inference. It can be seen, therefore, that the spatial correlation structure between the IMs plays a major role in the propagation of the observations to the grid points in the vicinity. Such a BN has the merit of providing probabilistic distributions of the ground-motion estimates, while ensuring that the joint distribution of the predicted parameters complies with the spatial correlation of the intraevent residuals.

Once the $Y_{i}$ distributions are obtained at the grid points, they may be interpolated at the locations of the vulnerable sites (e.g., built areas or infrastructure elements), while local amplification factors may also be added to account for site effects. The expression of the problem as a GBN has the merit of manipulating only continuous variables, which do not require a preliminary discretization and the creation of conditional probability tables that grow exponentially with the number of parents.

Single-IM Bayesian Inference on a Synthetic Example

A trivial synthetic example is introduced to demonstrate how the ground-motion map is updated with the $\mathrm{BN}$ approach. It consists of a $3 \times 3$ square grid (grid step $=1 \mathrm{~km})$ with an $M_{\mathrm{w}} 5.5$ earthquake occurring in its vicinity (at coordinates $[-3 ; 5]$ ), while two ground-motion records are assumed to be available (see the spatial configuration in Fig. 2a): the two observations (i.e., $Y_{\mathrm{obs} 1}$ and $Y_{\mathrm{obs} 2}$ ) are assumed to be $15 \%$ smaller and $10 \%$ larger than the predictions, respectively. For simplification purposes, only a single IM is considered here, which is the PGA estimated using the GMPE of Chiou and Youngs (2008).
The corresponding $\mathrm{BN}$ is detailed in Figure $2 \mathrm{~b}$, in which the link structure between $U_{i}$ and $Y(i)$ variables is characteristic of the triangular transformation matrix $\mathbf{T}$, following the Cholesky decomposition. This BN structure, consisting of a table describing the directed links between the variables and of normal distribution parameters for each variable (see equations 4 and 5), is then implemented in the Bayes Net toolbox (see Data and Resources). The junction tree algorithm, which carries out exact inference and thus provides exact probability distributions, is used within the toolbox. This algorithm consists in the following steps.

- Moralization of the BN: all edges are represented as undirected links, and all the parents of a same node are linked by a new undirected edge, if they were not previously linked.

- Variable elimination: each node is successively removed while its adjacent nodes are connected through additional undirected edges (i.e., fill-in edges), if they were not previously linked. Then, a clique is formed by the eliminated node and all its adjacent nodes.

- Once all variables have been eliminated, the cliques are assembled into a junction tree (see Fig. 3).

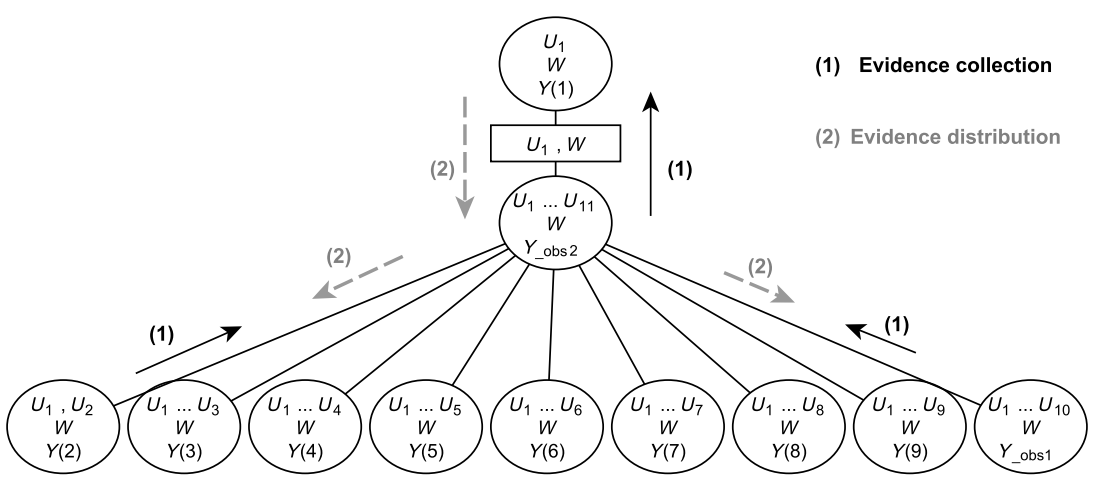

Figure 3. Junction tree corresponding to the example BN. Circles represent the cliques and the rectangular box is an example of a clique separator (i.e., set of nodes that are common to two connected cliques). The top circle is the root clique, and the bottom ones represent the leaves of the junction tree. 

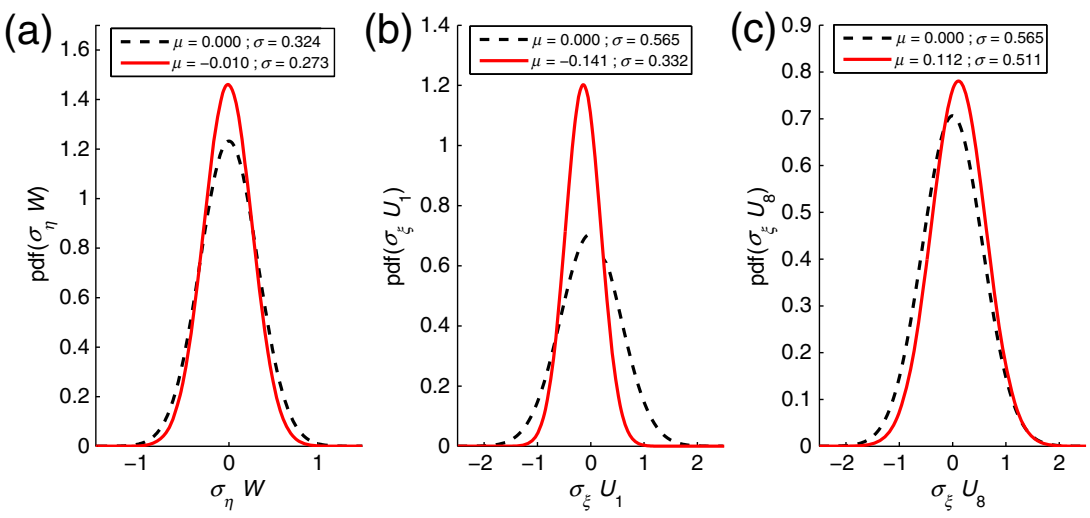

Figure 4. Prior (dashed line) and posterior (solid line) distributions for variables $W$, $U_{1}$, and $U_{8}$, representing (a) the interevent error, (b) an overestimated intraevent error, and (c) an underestimated intraevent error, respectively. The normal variables $U_{1}$ and $U_{8}$ have a strong link in the $\mathrm{BN}$ with the sites close to virtual stations numbers 1 and 2 , respectively. The color version of this figure is available only in the electronic edition. the $Y_{\mathrm{obs} 1}$ and $Y_{\mathrm{obs} 2}$ variables and propagated through the junction tree, as shown in Figure 3. The evidence propagation is carried out in two successive stages.

- Evidence collection: the evidence is collected from the leaves of the junction tree to the root clique. Operations of probability marginalization (i.e., removal of a variable) and multiplication are performed to update the potential of the root clique.

- Evidence distribution: the evidence is distributed from the root clique to all cliques along the junction tree. Operations of probability marginalization, division, and multiplication are performed to update the remaining cliques.
- The potential of each clique (i.e., joint probability distribution of the variables within the clique) is computed by multiplying the marginal and conditional Gaussian distributions that are associated with the variables (see equations 4 and 5).

Once the junction tree is built, the $\mathrm{BN}$ is considered as initialized, and it can be used to perform inference on any scenarios. In the proposed example, the evidence is set on

Table 1

Prior and Posterior Gaussian Distribution Parameters of the BN Variables Corresponding to the Synthetic Example

\begin{tabular}{|c|c|c|c|c|}
\hline \multirow[b]{2}{*}{ Variables } & \multicolumn{2}{|c|}{ Prior } & \multicolumn{2}{|c|}{ Posterior } \\
\hline & $\mu$ & $\sigma$ & $\mu$ & $\sigma$ \\
\hline$U_{1}$ & 0 & 1 & -0.2499 & 0.5877 \\
\hline$U_{2}$ & 0 & 1 & 0.0628 & 0.9667 \\
\hline$U_{3}$ & 0 & 1 & 0.1174 & 0.9667 \\
\hline$U_{4}$ & 0 & 1 & 0.0780 & 0.9674 \\
\hline$U_{5}$ & 0 & 1 & 0.1512 & 0.9379 \\
\hline$U_{6}$ & 0 & 1 & 0.1468 & 0.9498 \\
\hline$U_{7}$ & 0 & 1 & 0.1116 & 0.9720 \\
\hline$U_{8}$ & 0 & 1 & 0.1983 & 0.9050 \\
\hline$U_{9}$ & 0 & 1 & 0.1361 & 0.9564 \\
\hline$U_{10}$ & 0 & 1 & -0.1992 & 0.9194 \\
\hline$U_{11}$ & 0 & 1 & 0.1897 & 0.9126 \\
\hline$W$ & 0 & 1 & -0.0300 & 0.8434 \\
\hline$Y(1)$ & -1.6377 & 0.6508 & -1.7884 & 0.2163 \\
\hline$Y(2)$ & -1.5960 & 0.6508 & -1.6974 & 0.2976 \\
\hline$Y(3)$ & -1.5648 & 0.6508 & -1.6083 & 0.3906 \\
\hline$Y(4)$ & -1.6701 & 0.6508 & -1.7595 & 0.2884 \\
\hline$Y(5)$ & -1.6283 & 0.6508 & -1.6645 & 0.2762 \\
\hline$Y(6)$ & -1.5958 & 0.6508 & -1.5843 & 0.3325 \\
\hline$Y(7)$ & -1.7113 & 0.6508 & -1.7275 & 0.3591 \\
\hline$Y(8)$ & -1.6700 & 0.6508 & -1.6213 & 0.2418 \\
\hline$Y(9)$ & -1.6375 & 0.6508 & -1.5610 & 0.2528 \\
\hline$Y_{\mathrm{obs} 1}$ & -1.6335 & 0.6508 & -1.7961 & $\mathbf{0}$ \\
\hline$Y_{\mathrm{obs} 2}$ & -1.6526 & 0.6508 & -1.5573 & 0 \\
\hline
\end{tabular}

The numbers in bold correspond to the evidence from the observations. BN, Bayesian network.
The posterior probability distribution can then be observed for any variable of interest. For instance, the updated distribution of variable $Y(1)$ is obtained by marginalizing the potential of the clique $\left[U_{1} ; W ; Y(1)\right]$ with respect to $Y(1)$. The prior and posterior distribution parameters of the variables involved in the synthetic example are summarized in Table 1.

As expected, the ground-motion grid is modified by the field observations, that is, lower values are found toward the lower left of the grid at which the assumed observation Yobs 1 is lower than the initial prediction. An analysis of the distributions of the $\mathrm{BN}$ variables after the inference reveals two complementary levels of updating (Fig. 4).

- On a global level, the distribution of the $W$ variable, which represents the interevent error $\eta$ that is common to all grid points, is updated to provide a biased GMPE prediction that balances the general underestimation or overestimation of the ground motion when compared with the observations. In the present example, the two hypothetical ground-motion records are globally lower than the initial GMPE estimates with an unbiased interevent error: as a result, the variable $\eta$ is updated to account for the observed bias; the standard deviation $\sigma_{\eta}$ is also reduced, even though it does not converge toward zero due to the limited number of observations.

- On a local level, the distribution of the $U_{i}$ variables, which are used to map the spatially correlated intraevent errors $\zeta_{i}$, is updated to match the local variations of the ground motion in the vicinity of each of the two hypothetical stations. For instance, the closest grid point to observation number 1 is heavily influenced by the parent variable $U_{1}$ according to the corresponding element in the transformation matrix $\mathbf{T}$ (i.e., $t_{1,1}=1$ ). Therefore, the posterior distribution of $U_{1}$ is shifted toward the left to represent overestimation of PGA by the initial GMPE prediction when compared with the observation. The same effect is observed for the grid points close to observation number 2 , in which the recorded PGA is higher than the initial GMPE prediction: 
the distribution of $U_{8}$, which has a strong weight in the transformation matrix (i.e., $\left.t_{8,8}=0.488\right)$ with respect to grid point $Y(8)$, is therefore shifted toward the right.

\section{Comparison with Current Ground-} Motion Map Methods

The BN-updated ground-motion field is first compared with the ShakeMap algorithm (Worden and Wald, 2016), developed by the U.S. Geological Survey, which has proven its operational abilities to deliver ground-motion maps in near real time. The main principles of this algorithm are summarized as follows, in the case of a basic ground-motion map using strong-motion data only (i.e., no conversion between macroseismic intensity and ground-motion parameters).

- Removal of the potential site amplification factors from the observed ground motions (i.e., correction to rock site).

- Computation of the global bias introduced by the recorded ground motions with respect to the initial GMPE estimates, and use of a bias-adjusted GMPE for the prediction at the grid points. This adjustment is achieved by finding the $M_{\mathrm{w}}$ magnitude that reduces the errors between the observed and the predicted ground motions when the GMPE is evaluated for the adjusted magnitude.

- Interpolation of the observations to the grid points.

- At each grid point, updating ground motion through a weighted average between the bias-adjusted GMPE estimate and the observations (Worden et al., 2010). The GMPE estimate is weighted by the inverse of the variance provided by the GMPE, while each observation is weighted by the term $1 / \sigma_{\mathrm{obs}}^{2}$ (i.e., $\sigma_{\mathrm{obs}}$ is the standard deviation assigned to the observation-it increases with the distance between the observation and the grid point based on a correlation model).

- Application of potential site amplification factors at the grid points.

In the ShakeMap method, the total standard deviation associated with each grid point is obtained as a byproduct of the interpolation process:

$$
\sigma_{\ln \mathrm{PGA}}=\sqrt{\frac{1}{\frac{1}{\sigma_{\mathrm{GMPE}}^{2}}+\sum_{j=1}^{k} \frac{1}{\sigma_{\mathrm{obs}, j}^{2}}}}
$$

(Worden et al., 2010), in which $\sigma_{\mathrm{obs}, j}$ is the standard deviation assigned to the $j$ th observation and $\sigma_{\mathrm{GMPE}}$ is the standard deviation of the GMPE estimate. As stated by Worden et al. (2010), if enough observations are used to update the event magnitude, $\sigma_{\mathrm{GMPE}}$ may be taken as equal to $\sigma_{\zeta}$ (i.e., intraevent variability only), which has been assumed here.

To apply the ShakeMap algorithm, one must ensure that comparable correlation models are applied to both Shake-
Table 2

osterior Ground-Motion Parameters of the Synthetic Example, btained with the Three Methods (BN, ShakeMap, and Analytical Solution)

\begin{tabular}{ccccc}
\multicolumn{2}{c}{ BN Method } & & \multicolumn{2}{c}{ ShakeMap Method } \\
\cline { 1 - 2 } \cline { 5 - 5 } PGA $\left(\mathrm{m} / \mathrm{s}^{2}\right)$ & $\sigma_{\text {tot_lnPGA }}$ & & PGA $\left(\mathrm{m} / \mathrm{s}^{2}\right)$ & $\sigma_{\text {tot_ln PGA }}$ \\
\hline .1672 & 0.2163 & & 0.1710 & 0.2080 \\
0.1832 & 0.2976 & & 0.1858 & 0.2808 \\
0.2002 & 0.3906 & & 0.2004 & 0.3568 \\
0.1721 & 0.2884 & & 0.1732 & 0.2758 \\
0.1893 & 0.2762 & & 0.1891 & 0.2750 \\
0.2051 & 0.3325 & & 0.2020 & 0.3106 \\
0.1777 & 0.3591 & & 0.1763 & 0.3319 \\
0.1977 & 0.2418 & & 0.1959 & 0.2350 \\
0.2099 & 0.2528 & & 0.2040 & 0.2404 \\
$\eta$ & & & $\eta$ & \\
0.0097 & 0.2730 & & -0.0336 & 0.0000 \\
& & &
\end{tabular}

, peak ground acceleration.

Map and BN-based approaches (equation 2). In the ShakeMap method, the influence of the observations on the grid predictions is modeled by a weighting function $F=\sigma_{\mathrm{obs}} / \sigma_{\mathrm{GMPE}}$, which tends toward zero when the intersite distance is zero and toward infinity for large distances. However, it appears that using the influence distances that are advocated in the ShakeMap manual (i.e., $r_{\mathrm{ROI}}=10 \mathrm{~km}$ and $r_{\mathrm{MAX}}=15 \mathrm{~km}$ ) corresponds to correlation coefficients that are much larger than the ones generated by equation (2), with $b=13.5 \mathrm{~km}$. Some numerical tests have shown that, to yield comparable results, the weighting function should follow the same shape as the proposed correlation model, with an exponentially decreasing rate. The mathematical link between the weighting function $F$ and the correlation coefficient $\rho$ is investigated in the Appendix, in which equivalent models for both the ShakeMap and the BN approaches are presented.

On the other hand, the use of the spatial correlation matrix in the BN approach to update the intraevent and interevent error distributions is conceptually similar to the analytical resolution of a conditional multivariate normal distribution, as proposed by Vanmarcke (1983) and Stafford (2012). This method directly computes the means and standard deviations of the intraevent and interevent error terms through vector and matrix multiplications. Therefore, it is straightforward to implement and fast to execute. However, due to its analytical nature, it becomes less favorable when additional variables are introduced, such as secondary IMs, macroseismic observations (which do not have a known spatial correlation structure), or even additional sources of epistemic uncertainties (e.g., GMPE selection). Its practical use for the generation of ground-motion maps is thus less interesting in the present context: yet, comparing the $\mathrm{BN}$ results with this analytical solution in the case of a single-IM inference is the most efficient way to validate the $\mathrm{BN}$ method. All three methods are applied to the synthetic example, for updating PGA distributions across a $3 \times 3$ grid: the updated ground-motion values and their associated uncertainties are detailed in Table 2. 


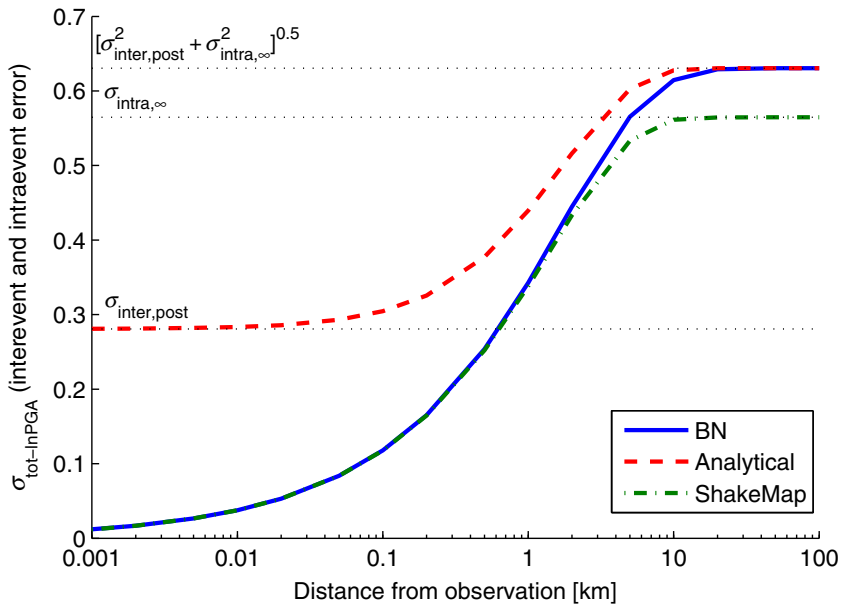

Figure 5. Evolution of the global error term (intraevent and interevent) as a function of the distance from an observation, for the three methods. $\sigma_{\text {inter,post }}$ represents the updated interevent standard deviation and $\sigma_{\text {intra, } \infty}$ represents the updated intraevent standard deviation very far from the observation (i.e., equivalent to the prior intraevent standard deviation). The color version of this figure is available only in the electronic edition.

Regarding the prediction of the ground-motion means, it appears first that the $\mathrm{BN}$ results are identical to the analytical solution, thus validating the accuracy of the proposed approach. The ShakeMap method, however, does not provide exactly the same means, which might be due to the way the updating is modeled, that is, through an interpolation instead of accounting for the full spatial correlation matrix. Moreover, both $\mathrm{BN}$ and analytical methods provide the same value for the interevent error term $\eta$ and its standard deviation, whereas the ShakeMap approach results in a lower value with zero uncertainty: this is explained by the bias removal through an optimization process, without accounting for the prior distribution and the associated likelihood function, as opposed to conditional updating methods.

On the other hand, the uncertainties in Table 2 are not exactly identical between the different methods, because the analytical method yields higher standard deviations than both the BN and ShakeMap methods, especially for grid points that are close to the observations. In the BN method, the global uncertainty appears to be sometimes lower than the interevent standard deviation $\sigma_{\eta}$, which is in contradiction to the widespread assumption of the quadratic combination of interevent and intraevent dispersions. To investigate this aspect, another synthetic example is considered, in which points are fixed along a line at increasing distances from a given observation, thus measuring the evolution of the ground-motion uncertainty with distance (see Fig. 5).

Many noteworthy observations can be made from this result.

1. Far from the observation, both the $\mathrm{BN}$ and analytical solutions converge to the same asymptote, which correspond to the quadratic combination of the updated interevent dispersion and the far-field intraevent dispersion.
Conversely, the global dispersion is underestimated by the ShakeMap method, which converges toward the intraevent dispersion only; this result is due to the assumption that sufficient observation points lead to an interevent term with zero dispersion (Worden et al., 2010), whereas the alternative methods have shown that this is not necessarily the case.

2. Close to the observation, both the BN and ShakeMap methods yield a global dispersion that tends toward zero. This behavior is consistent with a correlated Gaussian random field, in which predictions in the immediate vicinity of an observation are almost certain, with negligible dispersion. On the other hand, if the analytical solution is used, the dispersion tends toward the interevent standard deviation $\sigma_{\eta}$, which results in predictions that keep a significant dispersion even when very close to an observation. This discrepancy is explained by the following rationale.

- The analytical solution uses a two-step set of separate equations to compute the updated distributions of interevent and intraevent error terms.

- These terms may then be used to compute the global dispersion, thanks to a quadratic combination, under the assumption that the variables are independent.

- However, it appears that the intraevent error terms are dependent on the estimation of the interevent error, thus breaking the independency assumption and preventing the use of the quadratic combination. Therefore, although the analytical solution is perfectly valid for the separate estimation of interevent and intraevent error terms, it does not provide any means of accurately computing the global dispersion.

- The BN method, on the other hand, implicitly accounts for the correlation between the intraevent and interevent residuals through the multiplication of conditional probabilities: as a result, a stronger correlation close to an observation leads to a smaller global dispersion, which ultimately tends toward zero.

Therefore, the comparison between the different approaches has demonstrated that the $\mathrm{BN}$ method is as accurate as the analytical solution for updating mean values, whereas the ShakeMap method cannot provide the same values due to its interpolation scheme that is conceptually different from the use of spatial correlation models. Moreover, in terms of uncertainties, the BN method also provides the best solution to account for both the interevent and intraevent dispersions, whether the predictions are made close or far from an observation.

\section{Computational Performance}

The following subsections study the feasibility of the proposed approach for large spatial grids, while different strategies are investigated to facilitate its use in real-world applications. If such a method is to be used to develop an 
(a)

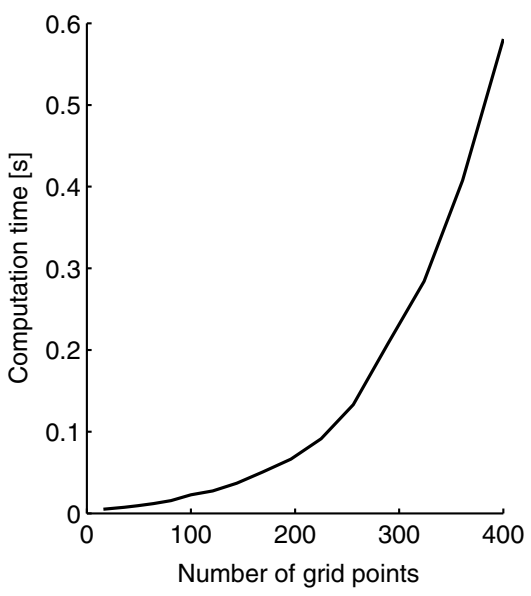

(b)

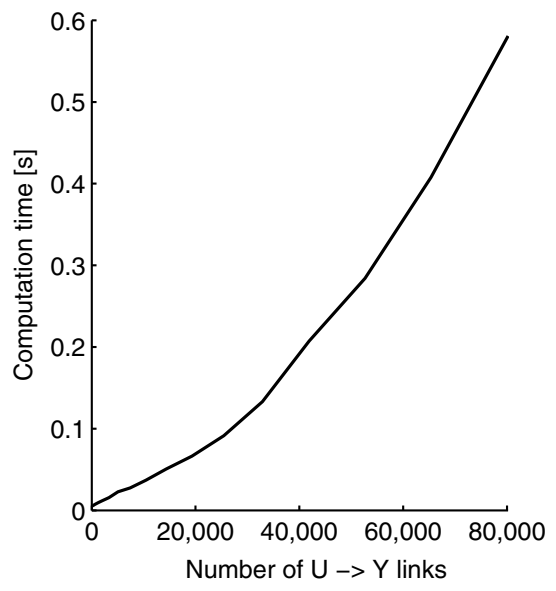

Figure 6. Computation time (on an Intel Core i5 processor with 4 GB RAM) for the Bayesian updating of one $\mathbf{Y}$ node, with respect to (a) the number of points in the grid and (b) the number of $\mathbf{U} \rightarrow \mathbf{Y}$ links in the corresponding BN.

operational decision support system, it has to be accurate enough (i.e., high-resolution grid) over a spatial extent that covers most of the earthquake's effects, thus possibly leading to a huge correlation matrix. Moreover, such a system is expected to deliver updated ground-motion fields almost immediately after the occurrence of an earthquake, to provide situational awareness to emergency responders.

\section{Scalability}

As stated above, the $\mathrm{BN}$ has been implemented in the Bayes Net toolbox, which enables the inference of GBNs through a junction tree algorithm. Thanks to the Gaussian formulation that enables the use of continuous variables, the computation time is expected to remain much lower than the same BN structure with discrete variables, which would lead to the creation of conditional probability tables and clique potentials with an intractable number of elements. The execution time of a single inference operation (i.e., updating of one $\mathbf{Y}$ node) is detailed in Figure 6 for different grid sizes, for a single IM prediction (i.e., no secondary IM). As expected, the computational load increases exponentially with the number of grid points, even if the execution time remains tractable for a large grid containing 400 points. In Figure 6b, the computation time is represented with respect to the number of $\mathbf{U} \rightarrow \mathbf{Y}$ links that are required in the BN. The almostlinear relation between these two indicators shows that they are closely related; therefore, the explosion in computational times is mostly due to the proposed BN formulation, which is associated with an exponential increase of links with respect to the number of nodes.

As a result, even with the use of GBNs, the proposed BN approach is eventually bound to reach its limits for very large grids, usually due to elongated computation times that no longer meet the demands of a near-real-time information system. This issue becomes especially pressing when

high-resolution maps are required, for example, grid steps around $1 \mathrm{~km}$ for areas spanning several hundreds of kilometers, which would lead to tens of thousands of grid points.

\section{Optimization Strategies}

To make the problem tractable, several optimized $\mathrm{BN}$ formulations for correlated Gaussian random fields have been proposed by Bensi et al. (2011b), who found that a numerical optimization of an approximate transformation matrix $\hat{\mathbf{T}}$ results in a better computational performance than a Cholesky decomposition. This optimization starts by specifying a number $m$ of $\mathbf{U}$ nodes to keep in the BN, so that the approximation of the correlated Gaussian random field can be expressed

as follows:

$$
\hat{\mathbf{Z}}=\hat{\mathbf{T}} \mathbf{U}_{m}+\mathbf{S V}
$$

in which $\hat{\mathbf{T}}$ is the approximated $n \times m$ transformation matrix, $\mathbf{V}$ is an $n \times 1$ vector of independent standard normal variables and it is multiplied by a diagonal $n \times n$ transformation matrix $\mathbf{S}$, for which the elements $s_{i}$ are used to correct the global variance of the variables in $\hat{\mathbf{Z}}$ :

$$
s_{i}=\sqrt{1-\sum_{k=1}^{m} \hat{t}_{i k}^{2}}
$$

This approximation may be seen as a generalization of a Dunnett-Sobel class of Gaussian random variables: the $\hat{t}_{i k}$ elements are found through a numerical optimization, with the objective of minimizing the difference between the actual correlation matrix $\mathbf{R}$ and its approximation $\hat{\mathbf{T}}^{t}$. Finally, once the transformation matrix has been optimized, further simplifications may be carried out, such as the removal of nodes (i.e., columns in $\hat{\mathbf{T}}$ ) or links (i.e., elements in $\hat{\mathbf{T}}$ ) from the BN: all these elimination strategies, based on importance measures checking the respective influence of each variable, are detailed in Bensi et al. (2011b). This strategy is tested on the synthetic example detailed above, with the aim of comparing its accuracy and computational efficiency. The scalability is also investigated by increasing the grid extent with different scenarios (see Table 3). The discrepancy between the exact solution (i.e., BN with Cholesky decomposition over the full grid) and the various approximations is measured with two metrics, namely the average of the absolute errors in predicting the PGA mean over the grid points and the maximum error.

It appears that the optimization strategy does not lead to any computational time gain, even at the cost of less accurate results (i.e., around $20 \%$ error rate on the prediction of the 


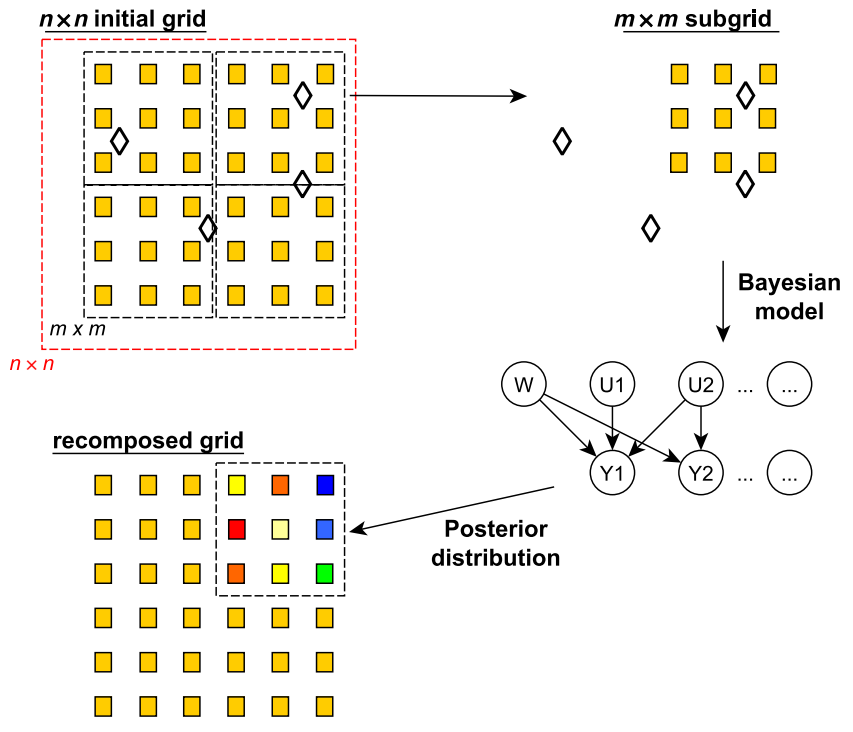

Figure 7. Illustration of the grid subdivision strategy, in which the diamonds represent observations. The color version of this figure is available only in the electronic edition.

mean PGA for larger grids). For smaller grids, the accuracy loss is negligible; however, as the number of grid points increases relatively to the number $m$, the quality of the approximation diminishes greatly, unless a very costly optimization is carried out with a large enough $m$ value. This observation is in strong contrast with the original findings by Bensi et al. (2011b), who have shown that this optimization significantly reduces the computational time while maintaining a reasonable accuracy. In the present concept, two main conceptual differences with the work by Bensi et al. (2011b) explain this difference.
- Continuous GBNs are implemented here instead of discrete $\mathrm{BNs}$, so that the initial computational bottlenecks (i.e., size of conditional probability table and cliques) are now removed to some extent and that the size of the correlation matrix is one of the main issues. The initialization time is listed in Table 3, thus revealing how difficult it is to perform the numerical optimization of a large matrix with a large number $m$ of variables. The BN inference time becomes less essential, although it should be noted that adding the $\mathbf{V}$ nodes has the effect of slightly increasing computational costs.

- The objective here is to perform a backward analysis (i.e., inference from an observation to other $\mathbf{Y}$ nodes), whereas only the accuracy of a forward analysis has been investigated in Bensi et al. (2011b). Backward analyses are more complex (i.e., message passing through many nodes) and they require a highly accurate correlation matrix.

Alternatively, a more radical and straightforward strategy is proposed in the present article, in which the initial grid containing $n \times n$ points is divided into $k$ subgrids of $m \times m$ points, in which $k=(n / m)^{2}$. As a result, $k$ BNs need to be created and solved before all the predictions at the grid points are aggregated and projected on the same map (Fig. 7).

As shown in Figure 7, all observations must be used as evidence within each BN to ensure that the updating of the variables is at the same level for each subgrid. This approach is justified by the fact that the BN inference appears to be robust with respect to the number and location of the $Y_{i}$ variables, as long as the evidence nodes remain unchanged (i.e., whatever the extent of the grid, the updated groundmotion field should remain stable). This grid subdivision

Table 3

Computational Cost and Accuracy of the Optimization Strategy, with Different Values of $m$ (Number of $\mathbf{U}$ Nodes)

\begin{tabular}{|c|c|c|c|c|c|c|}
\hline \multirow[b]{2}{*}{ Grid Size } & \multirow[b]{2}{*}{ Measure } & \multirow[b]{2}{*}{ Cholesky Decomposition } & \multicolumn{4}{|c|}{ Optimization } \\
\hline & & & $m=20$ & $m=10$ & $m=5$ & $m=2$ \\
\hline \multirow{4}{*}{$\begin{array}{c}3 \times 3 \\
(+2 \text { obs. })\end{array}$} & Mean error & Exact & N/A & $<0.1 \%$ & $0.1 \%$ & $0.5 \%$ \\
\hline & Maximum error & Exact & N/A & $<0.1 \%$ & $0.3 \%$ & $1.1 \%$ \\
\hline & Init. time & $<0.1 \mathrm{~s}$ & N/A & $7.7 \mathrm{~s}$ & $0.5 \mathrm{~s}$ & $0.2 \mathrm{~s}$ \\
\hline & BN time & $<0.1 \mathrm{~s}$ & N/A & $<0.1 \mathrm{~s}$ & $<0.1 \mathrm{~s}$ & $<0.1 \mathrm{~s}$ \\
\hline \multirow{4}{*}{$\begin{array}{c}6 \times 6 \\
(+4 \text { obs. })\end{array}$} & Mean error & Exact & $<0.1 \%$ & $0.4 \%$ & $2.6 \%$ & $7.7 \%$ \\
\hline & Maximum error & Exact & $0.2 \%$ & $1.0 \%$ & $6.1 \%$ & $13.2 \%$ \\
\hline & Init. time & $<0.1 \mathrm{~s}$ & $173.6 \mathrm{~s}$ & $20.5 \mathrm{~s}$ & $2.7 \mathrm{~s}$ & $0.6 \mathrm{~s}$ \\
\hline & BN time & $0.3 \mathrm{~s}$ & $0.9 \mathrm{~s}$ & $0.8 \mathrm{~s}$ & $0.7 \mathrm{~s}$ & $0.7 \mathrm{~s}$ \\
\hline \multirow{4}{*}{$\begin{array}{c}12 \times 12 \\
(+8 \text { obs. })\end{array}$} & Mean error & Exact & $1.4 \%$ & $2.2 \%$ & $3.3 \%$ & $3.6 \%$ \\
\hline & Maximum error & Exact & $6.3 \%$ & $9.9 \%$ & $13.1 \%$ & $12.8 \%$ \\
\hline & Init. time & $<0.1 \mathrm{~s}$ & $4237.6 \mathrm{~s}$ & $606.5 \mathrm{~s}$ & $117.6 \mathrm{~s}$ & $16.9 \mathrm{~s}$ \\
\hline & BN time & $5.9 \mathrm{~s}$ & $14.9 \mathrm{~s}$ & $14.1 \mathrm{~s}$ & $13.4 \mathrm{~s}$ & $12.9 \mathrm{~s}$ \\
\hline \multirow{4}{*}{$\begin{array}{c}24 \times 24 \\
(+16 \text { obs. })\end{array}$} & Mean error & Exact & Out of memory & Out of memory & Out of memory & $5.9 \%$ \\
\hline & Maximum error & Exact & Out of memory & Out of memory & Out of memory & $15.8 \%$ \\
\hline & Init. time & $<0.1 \mathrm{~s}$ & Out of memory & Out of memory & Out of memory & $1141.1 \mathrm{~s}$ \\
\hline & BN time & $1213.1 \mathrm{~s}$ & Out of memory & Out of memory & Out of memory & $2588.4 \mathrm{~s}$ \\
\hline
\end{tabular}

"Init. time" refers to the initialization time, corresponding to the construction of the transformation matrix $\mathbf{T}$ or its approximation $\hat{\mathbf{T}}$, "BN time" refers to the total duration of the Bayesian execution (i.e., construction of the junction tree and Bayesian inference for all grid points) on a standard PC. The mean and maximum error measures refer to the PGA mean value. 
Table 4

Computational Cost and Accuracy of the Grid Subdivision Strategy, with Different Subgrid Sizes, for the Large $24 \times 24$ Grid Tested in Table 3

\begin{tabular}{|c|c|c|c|c|c|c|}
\hline Measure & \multicolumn{2}{|c|}{$64 \times(3 \times 3)$ Subgrids } & \multicolumn{2}{|c|}{$16 \times(6 \times 6)$ Subgrids } & \multicolumn{2}{|c|}{$4 \times(12 \times 12)$ Subgrids } \\
\hline Init. time & \multicolumn{2}{|c|}{$<0.1 \mathrm{~s}$} & \multicolumn{2}{|c|}{$<0.1 \mathrm{~s}$} & \multicolumn{2}{|c|}{$<0.1 \mathrm{~s}$} \\
\hline BN time & \multicolumn{2}{|c|}{$10.3 \mathrm{~s}$} & \multicolumn{2}{|c|}{$8.8 \mathrm{~s}$} & \multicolumn{2}{|c|}{$27.9 \mathrm{~s}$} \\
\hline$d_{\text {corr }}=4.5 \mathrm{~km}$ & Mean PGA & $\sigma_{\text {tot_ln PGA }}$ & Mean PGA & $\sigma_{\text {tot_ln PGA }}$ & Mean PGA & $\sigma_{\text {tot_ln PGA }}$ \\
\hline Mean error & $2.14 \times 10^{-4} \%$ & $9.37 \times 10^{-4} \%$ & $1.81 \times 10^{-4} \%$ & $8.82 \times 10^{-4} \%$ & $1.57 \times 10^{-4} \%$ & $8.50 \times 10^{-4} \%$ \\
\hline $\begin{array}{l}\text { Maximum error } \\
d_{\text {corr }}=9 \mathrm{~km}\end{array}$ & $1.83 \times 10^{-3} \%$ & $4.33 \times 10^{-3} \%$ & $1.63 \times 10^{-3} \%$ & $4.41 \times 10^{-3} \%$ & $1.54 \times 10^{-3} \%$ & $3.72 \times 10^{-3} \%$ \\
\hline Mean error & $1.93 \times 10^{-4} \%$ & $7.51 \times 10^{-4} \%$ & $1.80 \times 10^{-4} \%$ & $7.80 \times 10^{-4} \%$ & $1.48 \times 10^{-4} \%$ & $7.29 \times 10^{-4} \%$ \\
\hline $\begin{array}{l}\text { Maximum error } \\
d_{\text {corr }}=18 \mathrm{~km}\end{array}$ & $2.13 \times 10^{-3} \%$ & $4.75 \times 10^{-3} \%$ & $3.53 \times 10^{-3} \%$ & $7.92 \times 10^{-3} \%$ & $1.84 \times 10^{-3} \%$ & $4.75 \times 10^{-3} \%$ \\
\hline Mean error & $1.52 \times 10^{-4} \%$ & $8.12 \times 10^{-4} \%$ & $1.41 \times 10^{-4} \%$ & $8.24 \times 10^{-4} \%$ & $1.42 \times 10^{-4} \%$ & $8.12 \times 10^{-4} \%$ \\
\hline Maximum error & $2.90 \times 10^{-3} \%$ & $1.32 \times 10^{-2} \%$ & $2.25 \times 10^{-3} \%$ & $8.45 \times 10^{-3} \%$ & $1.96 \times 10^{-3} \%$ & $9.46 \times 10^{-3} \%$ \\
\hline
\end{tabular}

The error measures, applied to both the mean PGA and global standard deviation, are computed with respect to the full grid solution. The accuracy is quantified for three correlation lengths $d_{\text {corr }}$, the first one corresponding to the standard case (i.e., $b=3 d_{\text {corr }}=13.5 \mathrm{~km}$ ).

may be seen as an extreme case of the numerical optimization detailed above, in the sense that grid points from two different subgrids are similar as nodes between which links have removed. However, the main difference lies in the fact that all observations are kept for all subgrids to maintain the same inference across all subgrids. This strategy is tested on the largest grid of the synthetic example (i.e., $24 \times 24$ grid with 16 observations), which is divided in different sets of subgrids: the average and maximum error measures are estimated for both the PGA mean and standard deviation (see Table 4).

The grid subdivision provides a dramatic decrease in computational times, while the accuracy of the estimations remains very high and stable, that is, always below $0.1 \%$ of maximum error. This preliminary observation is investigated further by checking the evolution of the error rate with the correlation length, defined as $d_{\text {corr }}=b / 3$ if the correlation model from equation (2) is used. The spatial extent of the subgrid with respect to the correlation distance appears to govern slightly the evolution of the error rate. For small correlation distances, there are almost no differences between the various grids; while greater error rates, albeit still very small, may be observed when the grid extent (i.e., its total dimension) becomes much smaller than the correlation distance. Globally, these small deviations from the initial grid appear to be negligible, especially when considering that such a strategy enables almost any map size and resolution to be handled, with few computational constraints. Additional overlapping subgrids might also be considered to correct any boundary effects; however, the present configuration, with standard correlation lengths (e.g., up to a couple of dozen kilometers) and 1-km grid steps, results in excellent accuracy, especially when compared with the much larger error rates obtained with the optimized transformation matrix. One significant caveat, however, is that the use of subgrids requires the construction of independent BNs, thus preventing the computation of joint statistics for locations that do not belong to the same subgrid; such a feature is essential in the context of infrastructure risk analysis (i.e., the presence of interdependent assets at various locations). It may be overlooked, however, if the main objective is to generate a ground-motion map following an earthquake.

\section{Application to the 14 April $2016 M_{\mathrm{w}} 6.2$ Kumamato Earthquake}

The inference abilities of the proposed BN approach are demonstrated in the following subsections, in which strongmotion data from the $M_{\mathrm{w}} 6.2$ earthquake that occurred near Kumamoto, Japan, on 14 April 2016 (this was the foreshock of the destructive $M_{\mathrm{w}} 7.0$ event that occurred two days later in the same region) are exploited. This earthquake was recorded by a dense network of strong-motion stations in the near field. Its smaller magnitude than the mainshock enables a point-source event to be assumed.

\section{Single-IM Bayesian Inference}

The $M_{\mathrm{w}} 6.2$ Kumamoto earthquake (see Data and Resources) was recorded by a total of 192 local strong-motion instruments. For demonstration purposes, a distributed ground-motion field is predicted across a $100 \times 100 \mathrm{~km}^{2}$ area, which contains 26 strong-motion observations (Table 5 and Fig. 8). In this section, the $\mathrm{BN}$ approach is first demonstrated for a single-IM prediction (PGA only), without the cross correlation with other IMs (e.g., response spectral ordinates). Therefore, only 25 observations are exploited, because the PGA from station number 8 was not available.

The prior ground-motion field is computed with the GMPE of Chiou and Youngs (2008), assuming a strikeslip-faulting mechanism and a depth to top of rupture $Z_{\mathrm{TOR}}=5 \mathrm{~km}$. Before the PGA observations are entered in the $\mathrm{BN}$, they are converted to rock conditions by removing 


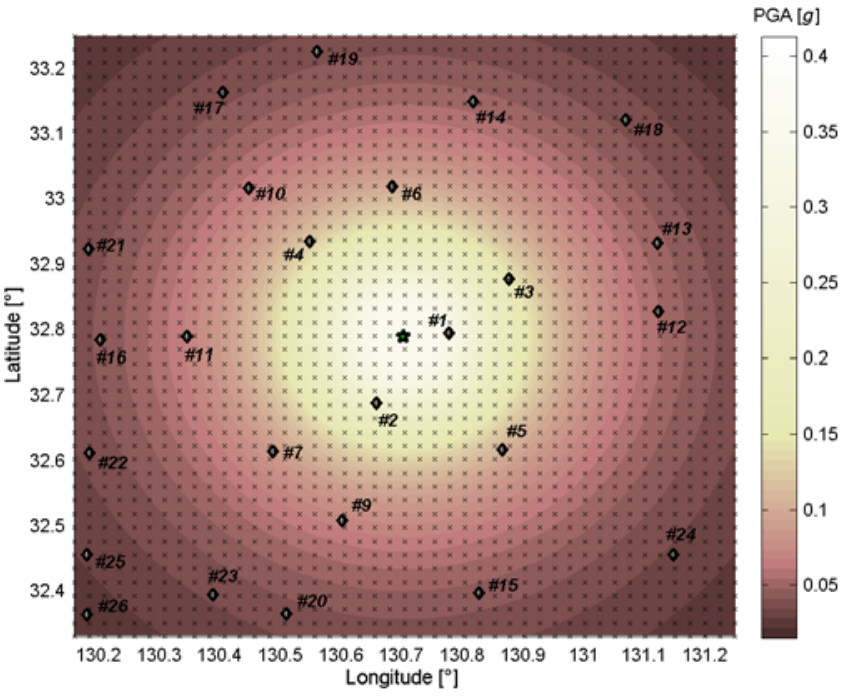

Figure 8. Prior estimation of peak ground acceleration $\left(\mathrm{PGA}_{\text {rock }}\right)$ using the source parameter and the ground-motion prediction equation (GMPE). The recording stations are represented by diamonds and the earthquake epicenter by a star. Small black crosses represent the 2025 grid points. The color version of this figure is available only in the electronic edition.

the amplification factors that are modeled in the GMPE using the time-averaged velocity of the top $30 \mathrm{~m} V_{S 30}$. The $V_{S 30}$ value for each seismic station is obtained from the K-NET database, whereas an extrapolation for profiles that are shallower than $30 \mathrm{~m}$ has been performed using the relationships provided by Boore et al. (2011).

A $48 \times 48$ global grid is used for the prediction of the ground motions, whereas a subdivision into sixteen $12 \times 12$ subgrids is adopted to reduce the computation time. As a result, the total number of points within each subgrid equals 144 (plus 25 observation points), which leads to a $169 \times 169$ correlation matrix. The resulting BN contains a total of 339 nodes and around 14,000 directed links between the variables.

By substituting $Y_{1, i}=\ln \mathrm{PGA}_{i}$ in equation (4) and by setting $\sigma_{\zeta}=0.518$ and $\sigma_{\eta}=0.296$ (i.e., intraevent and interevent standard deviation provided by Chiou and Youngs, 2008), the updated PGA field is computed using Bayesian inference (Fig. 9a). The spatial correlation model from equation (2) with $b=13.5 \mathrm{~km}$ for PGA is adopted here, although the choice of the spatial correlation model and its corresponding correlation distance remains a crucial issue and may have a large impact on the resulting ground-motion map, as noted by Crowley et al. (2008b). Other studies (e.g., Sokolov et al., 2010) have shown the significant variations in correlation lengths that may be deduced from different seismic arrays, even for the same geographical area. The total time taken for the generation of the ground-motion field is less than $3 \mathrm{~min}$ on a personal computer.

Table 5

Recording K-NET Stations Used and Corresponding PGA and Spectral acceleration (SA(1.0 s)) Values Corrected at a Rock Site, for the $M_{\mathrm{w}} 6.2$ Kumamoto Earthquake

\begin{tabular}{|c|c|c|c|c|c|c|}
\hline $\begin{array}{l}\text { Station } \\
\text { Number }\end{array}$ & Station ID & $\begin{array}{l}\text { Estimated } \\
V_{S 30}(\mathrm{~m} / \mathrm{s}) \\
\end{array}$ & $\begin{array}{c}\text { Recorded } \\
\operatorname{PGA}_{\text {rock }}\left(\mathrm{m} / \mathrm{s}^{2}\right) \\
\end{array}$ & $\begin{array}{l}\text { GMPE-Predicted } \\
\operatorname{PGA}_{\text {rock }}\left(\mathrm{m} / \mathrm{s}^{2}\right)\end{array}$ & $\begin{array}{c}\text { Recorded } \\
\mathrm{SA}(1.0 \mathrm{~s})_{\text {rock }}\left(\mathrm{m} / \mathrm{s}^{2}\right) \\
\end{array}$ & $\begin{array}{l}\text { GMPE-Predicted } \\
\mathrm{SA}(1.0 \mathrm{~s})_{\text {rock }}\left(\mathrm{m} / \mathrm{s}^{2}\right)\end{array}$ \\
\hline 1 & KMM006 & 195 & 4.03 & 3.26 & 1.93 & 1.42 \\
\hline 2 & KMM008 & 160 & 2.34 & 2.39 & 1.10 & 1.01 \\
\hline 3 & KMM005 & 287 & 1.34 & 1.46 & 0.64 & 0.60 \\
\hline 4 & КМM003 & 239 & 0.50 & 1.22 & 0.18 & 0.49 \\
\hline 5 & KMM011 & 185 & 2.66 & 1.05 & 0.23 & 0.43 \\
\hline 6 & KMM002 & 190 & 0.70 & 0.98 & 0.18 & 0.40 \\
\hline 7 & KMM010 & 149 & 0.38 & 0.86 & 0.25 & 0.35 \\
\hline 8 & КМM009 & 348 & - & - & 0.19 & 0.34 \\
\hline 9 & KMM012 & 205 & 0.75 & 0.70 & 0.35 & 0.29 \\
\hline 10 & NGS012 & 466 & 0.25 & 0.67 & 0.27 & 0.28 \\
\hline 11 & FKO016 & 363 & 0.44 & 0.63 & 0.11 & 0.26 \\
\hline 12 & КМM007 & 239 & 1.01 & 0.53 & 0.14 & 0.22 \\
\hline 13 & FKO014 & 858 & 0.34 & 0.49 & 0.17 & 0.21 \\
\hline 14 & КМM004 & 211 & 0.20 & 0.48 & 0.08 & 0.21 \\
\hline 15 & КMM014 & 641 & 0.57 & 0.44 & 0.07 & 0.19 \\
\hline 16 & NGS011 & 518 & 0.16 & 0.42 & 0.24 & 0.18 \\
\hline 17 & FKO015 & 134 & 0.42 & 0.38 & 0.16 & 0.17 \\
\hline 18 & KMM001 & 223 & 0.22 & 0.38 & 0.09 & 0.17 \\
\hline 19 & FKO013 & 259 & 0.37 & 0.38 & 0.08 & 0.16 \\
\hline 20 & КМM013 & 220 & 0.30 & 0.38 & 0.15 & 0.16 \\
\hline 21 & NGS008 & 547 & 0.18 & 0.37 & 0.11 & 0.16 \\
\hline 22 & NGS014 & 143 & 0.26 & 0.36 & 0.08 & 0.16 \\
\hline 23 & KMM018 & 287 & 0.29 & 0.35 & 0.03 & 0.16 \\
\hline 24 & MYZ020 & 256 & 0.44 & 0.33 & 0.06 & 0.15 \\
\hline 25 & КMМ019 & 490 & 0.26 & 0.28 & 0.03 & 0.13 \\
\hline 26 & KMM020 & 386 & 0.15 & 0.25 & 0.05 & 0.12 \\
\hline
\end{tabular}

GMPE, ground-motion prediction equation. 
(a)

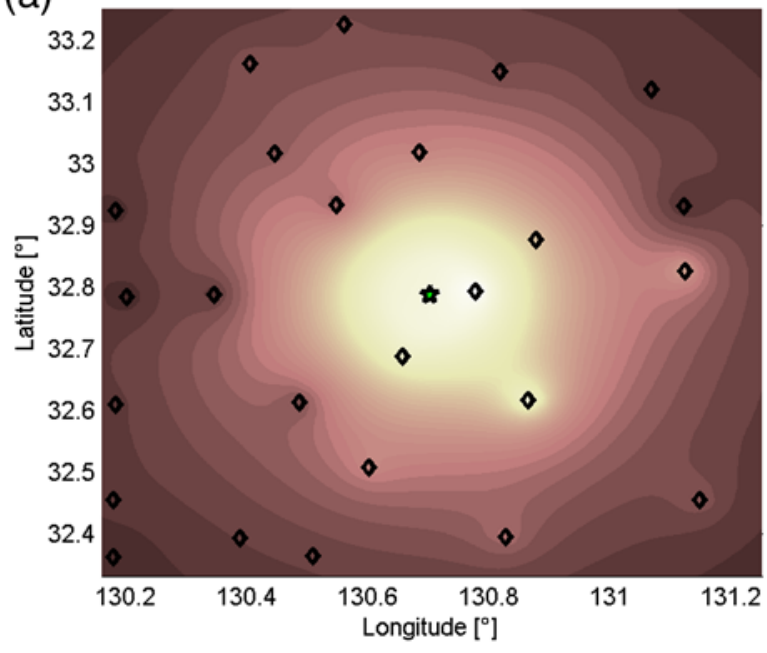

(b)

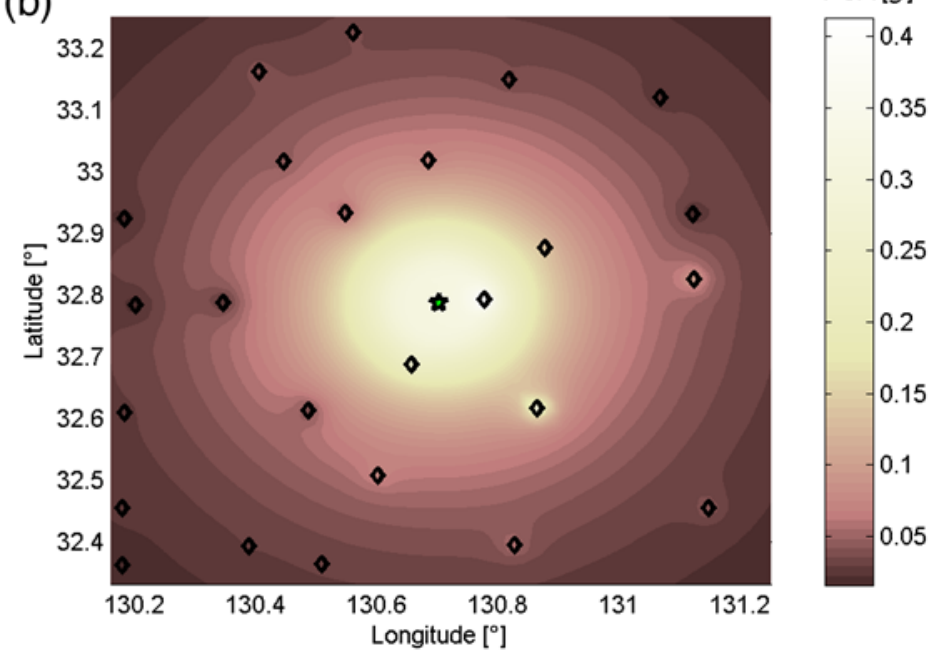

Figure 9. Updated shake map for $\mathrm{PGA}_{\text {rock }}$ using (a) the $\mathrm{BN}$ approach and (b) the ShakeMap algorithm. The recording stations are represented by diamonds and the earthquake epicenter by a star. The color version of this figure is available only in the electronic edition.

The updated ground-motion field from the ShakeMap method is also displayed in Figure 9b. It can be seen that the outcomes from both approaches are very similar; over all grid points, the averaged error rate between the ShakeMap and the $\mathrm{BN}$ results is $6.5 \%$, with a maximum of $32.2 \%$. There is a slight overestimation by the BN method, because the initial interevent variability of the GMPE (i.e., prior distribution) tends to constrain the updating of the distribution from the relatively small number of observations. Conversely, the ShakeMap algorithm has adjusted the event magnitude down to 5.952 to even out the global bias introduced by the observations. In the GMPE used, the relation between $M_{\mathrm{w}}$ and the $Y_{i}$ estimates is not linear, so lowering the magnitude is not exactly the same as lowering the interevent error. Other differences are due to the fact that the spatial correlation between grid points is not taken into account by the ShakeMap method, which relies on interpolation only, as discussed in the Demonstration of the Bayesian Network Approach section.

The total standard deviation of the PGA estimates by the two methods is also displayed in Figure 10. The results confirm the discussion in the Demonstration of the Bayesian Network Approach section (see Fig. 5). The dispersion of the predictions far from the observations is lower for the ShakeMap, due to the assumption that the interevent standard deviation can be set to zero if enough observations are present. On the contrary, the BN method provides an updated interevent standard deviation of 0.101 (instead of the initial value of 0.296), which has to be included in the field of intraevent dispersions. (a)

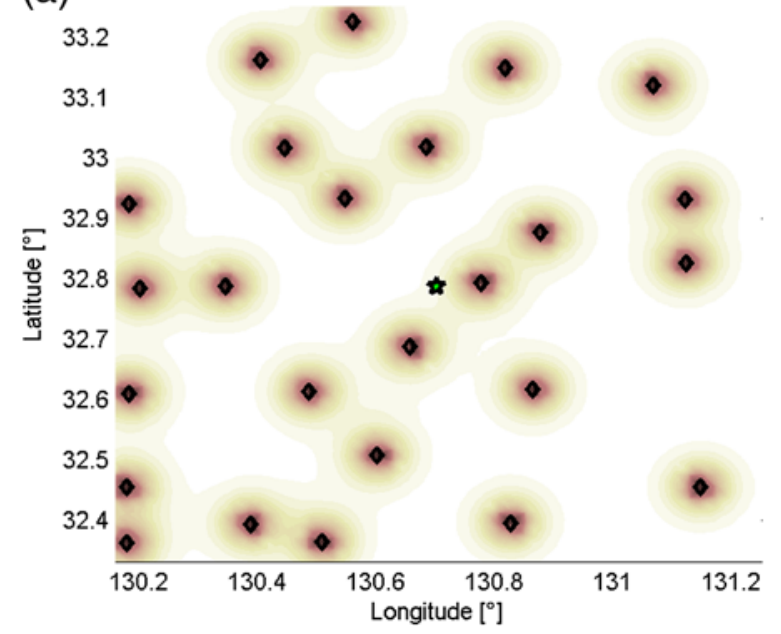

(b)

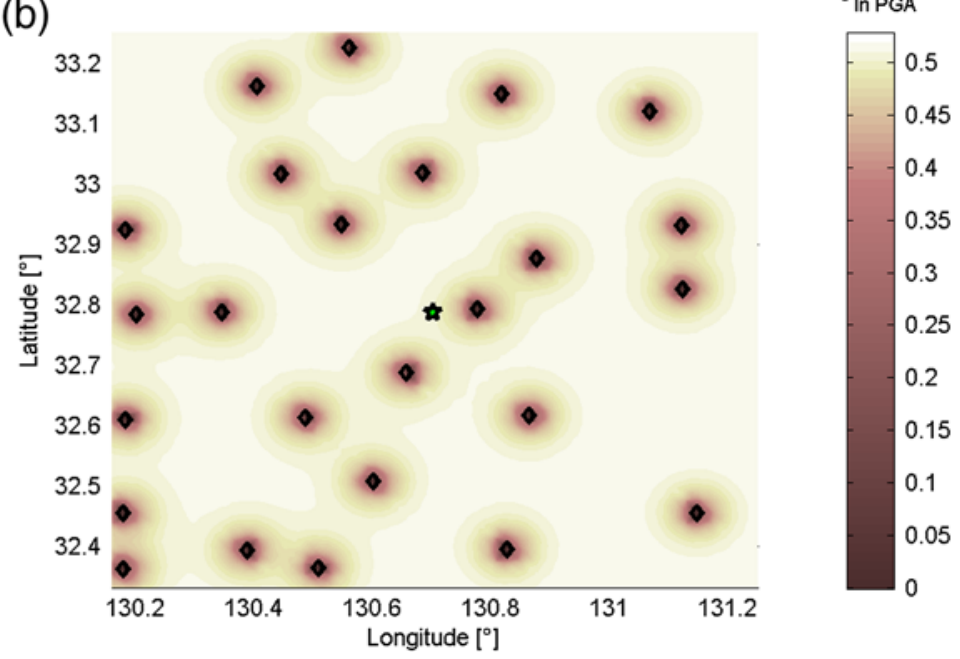

Figure 10. Updated field of $\sigma_{\ln \text { PGA }}$ using (a) the BN approach and (b) the ShakeMap algorithm. The color version of this figure is available only in the electronic edition. 
(a)

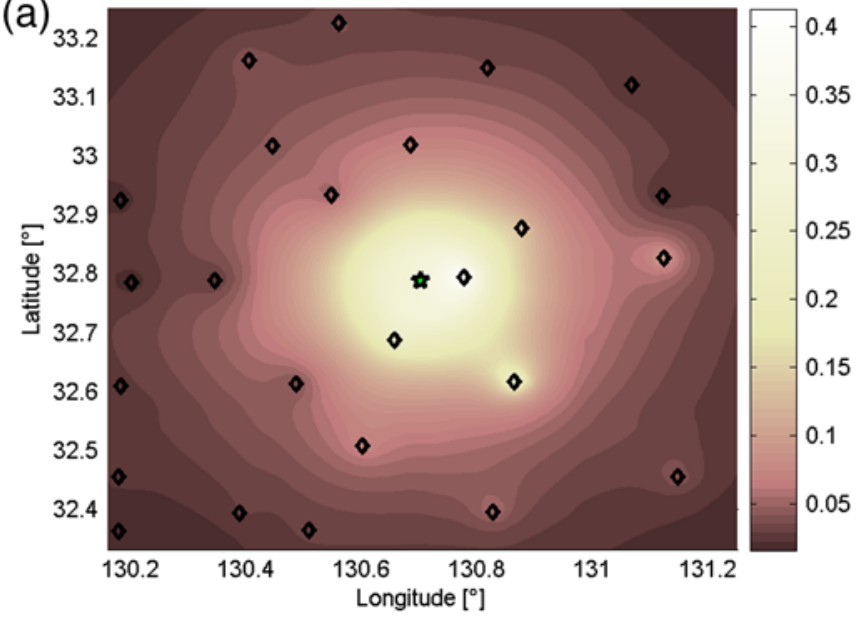

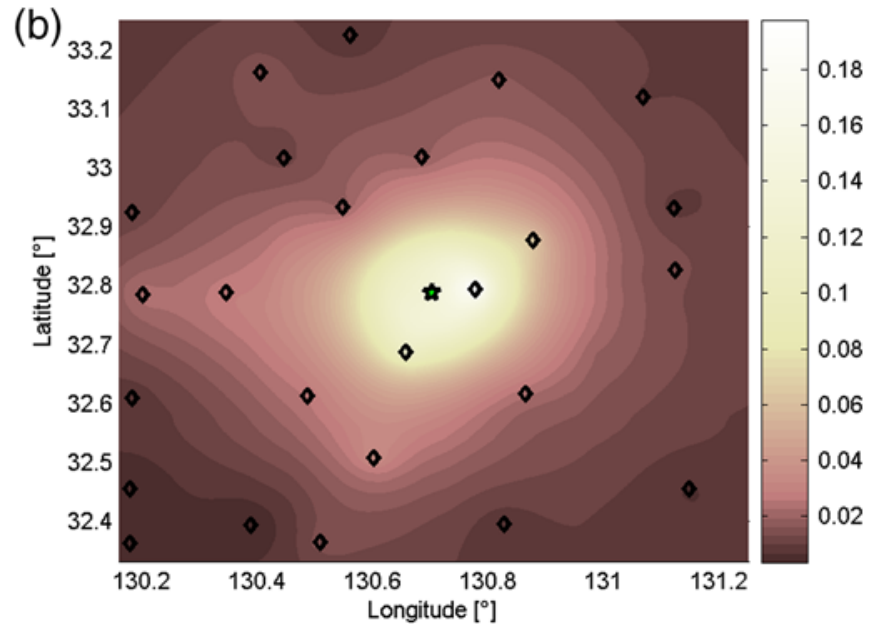

Figure 11. Updated $B N$-based shake map for (a) $\mathrm{PGA}_{\text {rock }}$ and (b) spectral acceleration (SA) rock at $1.0 \mathrm{~s}$ in $g$, using all observations from Table 5. Both sets of observations are used for the generation of each of the maps. The color version of this figure is available only in the electronic edition.

To summarize, the discrepancy in the estimation of the uncertainty fields derives from the way posterior distributions are computed in the $\mathrm{BN}$; the ground-motion inference relies entirely on updating the intraevent and interevent error terms, which are globally affected by the number and the spatial distribution of observations. On the other hand, the interpolation that is performed in the ShakeMap algorithm is strongly influenced by the observations in the immediate vicinity.

\section{Joint Inference on Two Cross-Correlated IMs}

The $M_{\mathrm{w}} 6.2$ Kumamoto earthquake is used again to demonstrate the inference of cross-correlated ground-motion fields, namely PGA as the primary IM and spectral acceleration $(\mathrm{SA}(1.0 \mathrm{~s}))$ as the secondary IM. Therefore, the vectorvalued ground-motion field may be updated from 25 PGAs and 26 values of SA(1.0 s), according to Table 5. Assuming a correlation distance of $20 \mathrm{~km}$ for $\mathrm{SA}(1.0 \mathrm{~s})$ and a period-toperiod cross-correlation coefficient of $\rho_{12}=0.587$ (Baker and Cornell, 2006), the inferred ground motions are displayed in Figure 11 for both cross-correlated IMs. It should be noted that another BN configuration has been tested, at which SA(1.0 s) becomes the primary IM and PGA the secondary one; the results are identical whatever the selected order of IMs, thanks to the message passing ability of BNs (i.e., the propagation of evidence is not necessarily influenced by the direction of the link between two variables).

Slight differences may be observed between the PGA field that has been estimated as a single-IM prediction (Fig. 9) and the one that is cross correlated with a secondary IM (Fig. 11). In particular, the PGA field appears to be altered at the location of station number 8 , which has no record of PGA, thanks to the contribution of the SA(1.0 s) observations, which provide additional constraints. This effect is demonstrated through a cross-validation study (see Table 6) on 25 stations for which PGA observations are available; for each station, the PGA observation is removed from the analysis and the prediction at this station's location. This process is repeated for three approaches, namely the ShakeMap method, the BN inference with PGA only, and the BN inference with both PGA and SA(1.0 s), to compare their predictive abilities.

The difference between the ShakeMap method and the single-IM BN inference is not very significant, as already suggested by the comparison of the respective ground-motion maps in the previous subsection. The multi-IM BN approach, however, introduces non-negligible changes in the PGA field and improves the prediction with respect to the observation in most cases. Aside from better constraining the ground-motion map, the ability of the BN approach to generate multiple IM fields is very useful for the rapid postearthquake damage assessment of different types of exposed assets.

\section{Integration of Macroseismic Intensities and Site Conditions}

To demonstrate the operational capabilities of the proposed $\mathrm{BN}$ approach, a ground-motion map is generated for a wider area, that is, a $200 \times 200 \mathrm{~km}^{2}$ surrounding the epicenter of the $M_{\mathrm{w}} 6.2$ Kumamoto foreshock, with a step grid of around $2 \mathrm{~km}$. Within this area, 90 strong-motion observations are found, along with 14 aggregated reports of macroseismic intensity. As with the ShakeMap algorithm, macroseismic data may be exploited in complement to strong-motion data, through the use of ground-motion intensity conversion equations (GMICEs; Wald et al., 1999). Starting from the BN in Figure 1, another set of BN nodes representing the macroseismic intensity is created, with a link pointing from each primary IM (i.e., PGA) node to each macroseismic intensity node. In the present example, the global GMICE developed by Caprio et al. (2015) has been used, thus the expression 
(a)

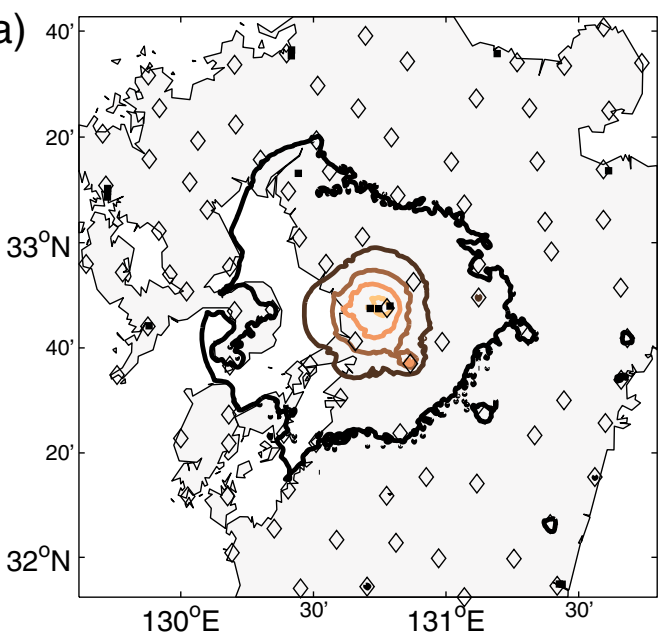

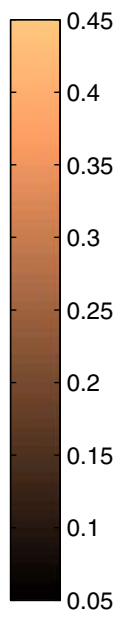

(b)

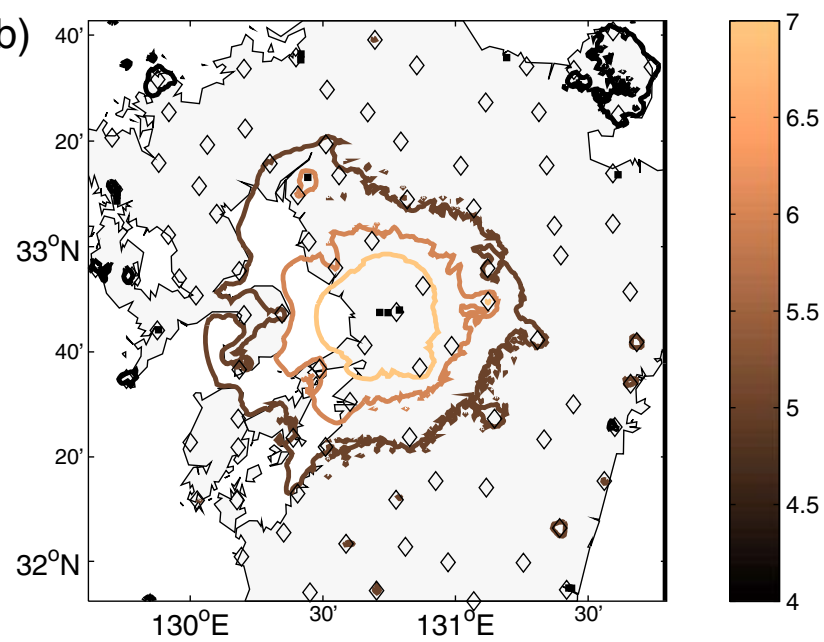

Figure 12. Updated BN-based shake map for (a) PGA in $g$ and (b) modified Mercalli intensity (MMI), accounting for site conditions and all available data (strong-motion data and macroseismic intensities). Strong-motion stations are represented by diamonds and intensity reports by black-filled squares. The color version of this figure is available only in the electronic edition.

of the modified Mercalli intensity (MMI) takes the following form:

$$
\mathrm{MMI}=\alpha+\beta \ln \mathrm{PGA}+\varepsilon_{\mathrm{MMI}},
$$

in which $\alpha$ and $\beta$ are GMICE coefficients, and $\varepsilon_{\text {MMI }}$ represents the error term of the regression, which follows a normal distribution with zero mean and standard deviation $\sigma_{\mathrm{MMI}}$.
Therefore, in the $\mathrm{BN}$, the conditional probability distribution of each $\mathrm{MMI}_{i}$ node, which is the child of a $Y_{1, i}$ node representing PGA, can be expressed as

$$
p\left(\mathrm{MMI}_{i} \mid Y_{1, i}\right)=N\left(\alpha+\beta Y_{1, i}, \sigma_{\mathrm{MMI}}^{2}\right) .
$$

As a result, the $\mathrm{BN}$ is able to collect evidence from various sources and pass the inference message in a two-way

Table 6

Updated Prediction of PGA for the 25 Stations, When Sequentially Removing the PGA Observation at the Given

\begin{tabular}{|c|c|c|c|c|c|c|}
\hline \multirow[b]{2}{*}{ Station Number } & \multicolumn{2}{|c|}{ ShakeMap } & \multicolumn{2}{|c|}{ BN (PGA only) } & \multicolumn{2}{|c|}{$\mathrm{BN}$ (PGA and SA) } \\
\hline & $\mathrm{PGA}_{\text {rock }}\left(\mathrm{m} / \mathrm{s}^{2}\right)$ & Prediction Error $(\%)$ & $\mathrm{PGA}_{\text {rock }}\left(\mathrm{m} / \mathrm{s}^{2}\right)$ & Prediction Error $(\%)$ & $\mathrm{PGA}_{\text {rock }}\left(\mathrm{m} / \mathrm{s}^{2}\right)$ & Prediction Error $(\%)$ \\
\hline 1 & 2.95 & -27.0 & 2.68 & -33.4 & 3.46 & -14.2 \\
\hline 2 & 2.11 & -9.7 & 1.97 & -15.8 & 2.21 & -5.8 \\
\hline 3 & 1.25 & -7.1 & 1.22 & -9.4 & 1.34 & -0.4 \\
\hline 4 & 1.03 & 107.2 & 1.00 & 102.5 & 0.64 & 29.2 \\
\hline 5 & 0.88 & -66.9 & 0.83 & -68.7 & 0.67 & -74.7 \\
\hline 6 & 0.81 & 16.0 & 0.79 & 12.1 & 0.58 & -17.3 \\
\hline 7 & 0.72 & 87.8 & 0.73 & 90.8 & 0.65 & 70.1 \\
\hline 9 & 0.57 & -23.9 & 0.56 & -25.5 & 0.69 & -8.1 \\
\hline 10 & 0.55 & 123.2 & 0.54 & 119.7 & 0.57 & 132.5 \\
\hline 11 & 0.51 & 17.9 & 0.50 & 15.5 & 0.37 & -15.3 \\
\hline 12 & 0.43 & -57.5 & 0.40 & -60.0 & 0.36 & -64.1 \\
\hline 13 & 0.40 & 15.4 & 0.41 & 17.8 & 0.40 & 15.5 \\
\hline 14 & 0.39 & 98.9 & 0.43 & 119.0 & 0.29 & 44.7 \\
\hline 15 & 0.35 & -37.9 & 0.36 & -37.2 & 0.24 & -57.6 \\
\hline 16 & 0.33 & 100.6 & 0.33 & 99.5 & 0.41 & 147.5 \\
\hline 17 & 0.30 & -28.3 & 0.31 & -26.7 & 0.34 & -18.2 \\
\hline 18 & 0.30 & 35.2 & 0.31 & 40.2 & 0.25 & 13.2 \\
\hline 19 & 0.30 & -19.3 & 0.31 & -16.7 & 0.22 & -40.1 \\
\hline 20 & 0.30 & -1.9 & 0.31 & 1.6 & 0.33 & 8.6 \\
\hline 21 & 0.29 & 63.9 & 0.30 & 67.8 & 0.25 & 40.4 \\
\hline 22 & 0.28 & 7.1 & 0.29 & 10.1 & 0.21 & -18.6 \\
\hline 23 & 0.28 & -2.8 & 0.29 & 0.2 & 0.14 & -52.1 \\
\hline 24 & 0.26 & -40.8 & 0.26 & -39.6 & 0.19 & -57.3 \\
\hline 25 & 0.22 & -15.9 & 0.23 & -14.9 & 0.13 & -50.7 \\
\hline 26 & 0.19 & 24.0 & 0.21 & 33.2 & 0.17 & 10.8 \\
\hline
\end{tabular}
Station

The prediction error measures the relative error rate with the actual observation. 
manner, that is, (1) from a PGA observation up to the neighboring grid points and finally to the converted intensities on the grid or (2) from the reported intensity up to the converted PGA at the same location and finally to the neighboring grid points. The generated ground-motion maps for both PGA and MMI are displayed in Figure 12, after a site correction has been applied to the inferred variables at rock conditions.

\section{Conclusions}

The BN formulation presented in this article, which makes use of the spatial distribution of the intraevent and interevent errors in the GMPE, has been successfully tested on a real-world example, thus validating the way the groundmotion inference is performed in the proposed Bayesian framework. Therefore, such a result lays a solid foundation for the development of more elaborate BNs that integrate damage and loss assessments, which may be used as part of an operational decision support system for emergency responders.

The comparison with the ShakeMap algorithm has provided valuable lessons on the respective merits of each approach. Although computationally costlier, the BN method offers a different philosophy when treating uncertainties because a more refined estimation of the posterior distribution of the interevent error is possible. Such an approach may be imagined in complement to the current ShakeMap algorithm, to adjust the value of interevent error with respect to the number of observations, for instance. On the other hand, there is no obvious link between the weighted interpolation used in the ShakeMap algorithm and the spatial correlation coefficient used in the BN method, which complicates the direct comparison of the two approaches. However, the analysis and the comparison of maps generated with these two complementary approaches could be useful to help constrain the current correlation models.

Moreover, the ability of the BN approach to compute vector-valued IM fields and to access the joint probabilities of IMs across several locations should prove highly beneficial when dealing with the loss prediction of infrastructure systems, for which the components are often susceptible to different IMs. Such inferences come at a high computational cost, which are currently not suitable for the nearreal-time applications that are covered by the ShakeMap framework. Conversely, in the case of the risk management of spatially distributed infrastructure systems, in which the GMPE has to be carried out for a limited number of sites, the BN approach might provide a rigorous probabilistic framework for the rapid loss assessment of interdependent components.

Finally, the proposed BN has mainly been focused on the treatment of aleatory variabilities (i.e., GMPE error terms); however, other variables representing epistemic uncertainties may be added to the BN, such as different GMPE candidates or different source or site assumptions. Provided that sufficient field observations are gathered, the $\mathrm{BN}$ inference would then be able to better constrain these parameters.

\section{Data and Resources}

The metadata on the $M_{\mathrm{w}} 6.2$ Kumamoto earthquake for the generation of the ground-motion map have been taken from the U.S. Geological Survey ShakeMap webpage (http://earthquake.usgs.gov/earthquakes/eventpage/us20005h zn\#shakemap, last accessed August 2017). The information on the soil profiles of the seismic stations has been taken from the K-NET network webpage (http://www.kyoshin.bosai.go .jp/, last accessed August 2017). The Bayes Net toolbox has been written by Kevin Murphy, and it is available from the webpage https://github.com/bayesnet/bnt (last accessed August 2017).

\section{Acknowledgments}

This research has been partially supported by the internal research program PSO VULNERABILITE at Bureau de Recherches Géologiques et Minières (BRGM), France, and by the European Commission's FP7 project INFRARISK (Grant Agreement Number 603960) at University College London, United Kingdom. The authors thank two anonymous reviewers for their detailed and careful comments that led to significant improvements to this study.

\section{References}

Baker, J. W., and C. A. Cornell (2006). Correlation of response spectral values for multicomponents ground motions, Bull. Seismol. Soc. Am. 96, no. 1, 215-227.

Bensi, M., A. Der Kiureghian, and D. Straub (2011a). A Bayesian network methodology for infrastructure seismic risk assessment and decisionsupport, PEER Report 2011/02, Pacific Earthquake Engineering Research Center, University of California, Berkeley, California.

Bensi, M., A. Der Kiureghian, and D. Straub (2011b). Bayesian network modeling of correlated random variables drawn from a Gaussian random field, Struct. Saf. 33, no. 6, 317-332.

Bensi, M., A. Der Kiureghian, and D. Straub (2013). Efficient Bayesian network modeling of systems, Reliab. Eng. Syst. Saf. 112, 200-213.

Boore, D. M., E. M. Thompson, and H. Cadet (2011). Regional correlations of $V_{S 30}$ and velocities averaged over depths less than and greater than 30 meters, Bull. Seismol. Soc. Am. 106, no. 6, 3046-3059.

Cavalieri, F., P. Franchin, P. Gehl, and D. D'Ayala (2017). Bayesian networks and infrastructure systems: Computational and methodological challenges, in Risk and Reliability Analysis: Theory and Applications, P. Gardoni (Editor), Springer, Cham, Switzerland, 385-415.

Caprio, M., B. Tarigan, B. C. Worden, S. Wiemer, and D. J. Wald (2015). Ground motion to intensity conversion equations (GMICEs): A global relationship and evaluation of regional dependency, Bull. Seismol. Soc. Am. 105, no. 3, 1476-1490.

Chiou, B. S., and R. R. Youngs (2008). An NGA model for the average horizontal component of peak ground motion and response spectra, Earthq. Spectra 24, no. 1, 173-215.

Crowley, H., and J. J. Bommer (2006). Modelling seismic hazard in earthquake loss models with spatially distributed exposure, Bull. Earthq. Eng. 4, no. 3, 249-273.

Crowley, H., J. J. Bommer, and P. J. Stafford (2008a). Recent developments in the treatment of ground-motion variability in earthquake loss models, J. Earthq. Eng. 12, no. 2, 71-80. 
Crowley, H., J. J. Bommer, and P. J. Stafford (2008b). Can earthquake loss models be validated using field observations? J. Earthq. Eng. 12, no. 7, $1078-1104$.

Douglas, J. (2007). Inferred ground motions on Guadeloupe during the 2004 Les Saintes earthquake, Bull. Earthq. Eng. 5, no. 3, 363-376.

Douglas, J., and B. Edwards (2016). Recent and future developments in earthquake ground motion estimation, Earth Sci. Rev. 160, 203-219.

Ebel, J. E., and D. J. Wald (2003). Bayesian estimations of peak ground acceleration and 5\% damped spectral acceleration from modified Mercalli intensity data, Earthq. Spectra 19, no. 3, 511-529.

Erdik, M., K. Şeşetyan, M. B. Demircioğlu, U. Hancılar, and C. Zülfikar (2011). Rapid earthquake loss assessment after damaging earthquakes, Soil Dynam. Earthq. Eng. 31, no. 2, 247-266.

Esposito, S., and I. Iervolino (2011). PGA and PGV spatial correlation models based on European multievent datasets, Bull. Seismol. Soc. Am. 101, no. 5, 2532-2541.

Gehl, P., F. Cavalieri, P. Franchin, and C. Negulescu (2017). Robustness of a hybrid simulation-based/Bayesian approach for the risk assessment of a real-world road network, Proc. of the 12th International Conf. on Structural Safety and Reliability, Vienna, Austria, 6-10 August 2017.

Jaiswal, K., D. J. Wald, and D. D'Ayala (2011). Developing empirical collapse fragility functions for global building types, Earthq. Spectra 27, no. 3, 775-795.

Jayaram, N., and J. W. Baker (2009). Correlation model for spatially distributed ground-motion intensities, Earthq. Eng. Struct. Dynam. 38, no. 15, 1687-1708.

Murphy, K. P. (2002). Dynamic Bayesian networks: representation, inference and learning, Ph.D. Dissertation, University of California, Berkeley.

Oliver, D. S. (2003). Gaussian cosimulation: Modelling of the crosscovariance, Math. Geol. 356, 681-698.

Park, J., P. Bazzurro, and J. W. Baker (2007). Modeling spatial correlation of ground motion intensity measures for regional seismic hazard and portfolio loss estimation, Proc. of the 10th International Conf. on Applied Statistics and Probability, Tokyo, Japan, 31 July-3 August 2007.

Pozzi, M., and A. Der Kiureghian (2013). Gaussian Bayesian network for reliability analysis of a system of bridges, Proc. of the 11th International Conf. on Structural Safety and Reliability, New York, New York, 16-20 June 2013.

Sokolov, V., F. Wenzel, W. Y. Jean, and K. L. Wen (2010). Uncertainty and spatial correlation of earthquake ground motion in Taiwan, Terr. Atmos. Ocean. Sci. 21, no. 6, 905-921.

Stafford, P. J. (2012). Evaluation of structural performance in the immediate aftermath of an earthquake: A case study of the 2011 Christchurch earthquake, Int. J. Forensic Eng. 1, no. 1, 58-77.

Vanmarcke, E. (1983). Random Fields, Analysis and Synthesis, The MIT Press, Cambridge, Massachusetts.

Wald, D. J., K. W. Lin, K. Porter, and L. Turner (2008). ShakeCast: Automating and improving the use of ShakeMap for post-earthquake decision-making and response, Earthq. Spectra 24, no. 2, 533-553.

Wald, D. J., V. Quitoriano, T. H. Heaton, and H. Kanamori (1999). Relationships between peak ground acceleration, peak ground velocity and modified Mercalli intensity in California, Earthq. Spectra 15, no. 3, 557-564.

Wald, D. J., B. C. Worden, V. Quitoriano, and K. L. Pankow (2005). ShakeMap manual: Technical manual, user's guide, and software guide, U.S. Geol. Surv. Tech. Methods 12-A1, $132 \mathrm{pp}$.

Weatherill, G., S. Esposito, I. Iervolino, P. Franchin, and F. Cavalieri (2014). Framework for seismic hazard analysis of spatially distributed systems, in SYNER-G: Systemic Seismic Vulnerability and Risk Assessment of Complex Urban, Utility, Lifeline Systems and Critical Facilities-Methodology and Applications, K. Pitilakis, P. Franchin, B. Khazai, and H. Wenzel (Editors), Springer, Dordrecht, The Netherlands, 57-88.

Worden, B. C., and D. J. Wald (2016). ShakeMap Manual Online: Technical Manual, User's Guide, and Software Guide, available at http://usgs .github.io/shakemap (last accessed July 2017).
Worden, B. C., D. J. Wald, T. I. Allen, K. W. Lin, D. Garcia, and G. Cua (2010). A revised ground-motion and intensity interpolation scheme for ShakeMap, Bull. Seismol. Soc. Am. 100, no. 6, 3083-3096.

\section{Appendix}

The objective of this Appendix is to establish a correspondence between the weighting function used in the ShakeMap interpolation and the spatial correlation coefficient used in the BN approach.

Consider two independent and identically distributed normal variables $X$ and $Y$, in which $X$ represents the initial GMPE estimate at a given grid point and $Y$ represents an observation at a distance $r$.

According to Worden et al. (2010), the interpolated value $X_{\text {int }}$ at the grid point may be computed from the following expression:

$$
X_{\mathrm{int}}=\frac{\frac{X}{\sigma_{X}^{2}}+\frac{Y}{\sigma_{Y}^{2}}}{\frac{1}{\sigma_{X}^{2}}+\frac{1}{\sigma_{Y}^{2}}}=\frac{\frac{X}{\sigma_{X}^{2}}+\frac{Y}{\sigma_{X}^{2} F(r)^{2}}}{\frac{1}{\sigma_{X}^{2}}+\frac{1}{\sigma_{X}^{2} F(r)^{2}}}=\frac{X+\frac{Y}{F(r)^{2}}}{1+\frac{1}{F(r)^{2}}}=\frac{F(r)^{2} X+Y}{F(r)^{2}+1},
$$

in which $F(r)=\sigma_{Y} / \sigma_{X}$ is the weighting function defined by Worden et al. (2010), and $X_{\text {int }}$ is assumed to have the following standard deviation:

$$
\sigma_{X_{\mathrm{int}}}=\sqrt{\frac{1}{\frac{1}{\sigma_{X}^{2}}+\frac{1}{\sigma_{X}^{2} F(r)^{2}}}}=\frac{\sigma_{X} F(r)}{\sqrt{F(r)^{2}+1}} .
$$

According to the above definitions, the correlation coefficient between the variables $Y$ and $X_{\text {int }}$ must correspond to the spatial correlation coefficient $\rho$ between two sites separated by a distance $r$. Therefore we can write:

$$
\begin{aligned}
\rho(r)= & \frac{\operatorname{cov}\left(X_{\mathrm{int}}, Y\right)}{\sigma_{X_{\text {int }}} \sigma_{Y}} \\
= & \frac{1}{\sigma_{X_{\text {int }}} \sigma_{Y}\left(F(r)^{2}+1\right)} \operatorname{cov}\left(F(r)^{2} X+Y, Y\right) \\
= & \frac{F(r)^{2}}{\sigma_{X_{\text {int }}} \sigma_{Y}\left(F(r)^{2}+1\right)} \operatorname{cov}(X, Y) \\
& +\frac{1}{\sigma_{X_{\text {int }}} \sigma_{Y}\left(F(r)^{2}+1\right)} \operatorname{cov}(Y, Y) .
\end{aligned}
$$

By definition, we have $\operatorname{cov}(Y, Y)=\sigma_{Y}^{2}$ and $\operatorname{cov}(X, Y)=0$, due to the independence assumption. Therefore, the expression of the spatial correlation coefficient becomes:

$$
\begin{aligned}
\rho(r) & =\frac{\sigma_{Y}}{\sigma_{X_{\mathrm{int}}}\left(F(r)^{2}+1\right)}=\frac{\sigma_{X} F(r)}{\sigma_{X_{\mathrm{int}}}\left(F(r)^{2}+1\right)} \\
& =\frac{1}{\sqrt{F(r)^{2}+1}} .
\end{aligned}
$$




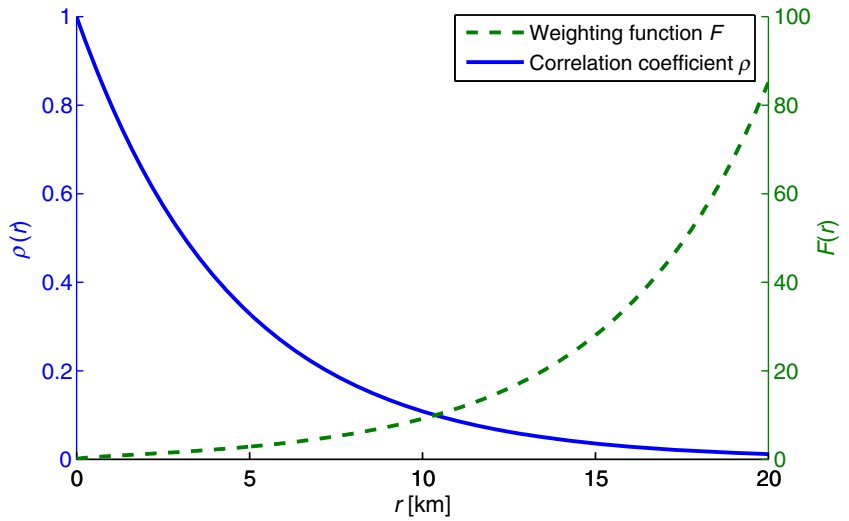

Figure A1. Adopted spatial correlation model and weighting function, for $b=13.5 \mathrm{~km}$. The color version of this figure is available only in the electronic edition.

If a spatial correlation model with an exponential decrease rate is used here (i.e., see equation 8), then the weighting function $F(r)$ that is proposed for the ShakeMap algorithm becomes:

$$
F(r)=\sqrt{\frac{1}{\rho^{2}}-1}=\sqrt{\left[\exp \left(\frac{3 r}{b}\right)\right]^{2}-1}
$$

in which $b$ is the correlation length.
The evolution of the weighting function $F(r)$ and of the correlation coefficient $\rho(r)$ with respect to intersite distance $r$ is represented in Figure A1, for $b=13.5 \mathrm{~km}$.

Bureau de Recherches Géologiques et Minières/

Direction Risques et Prévention (BRGM/DRP)

3 avenue C. Guillemin, BP 36009

45060 Orléans Cedex 2

France

p.gehl@brgm.fr

(P.G.)

Department of Civil and Environmental Engineering

University of Strathclyde

James Weir Building, 75 Montrose Street

Glasgow G11XJ, United Kingdom

(J.D.)

Department of Civil, Environmental and Geomatic Engineering University College London

Gower Street

London WC1E 6BT, United Kingdom (D.D.)

Manuscript received 7 March 2017;

Published Online 26 September 2017 